\title{
Diseases, lesions and malformations in the long-beaked common dolphin Delphinus capensis from the Southeast Pacific
}

\author{
Marie-Françoise Van Bressem ${ }^{1,6, *}$, Koen Van Waerebeek ${ }^{1}$, David Montes ${ }^{1}$, \\ Seamus Kennedy ${ }^{2}$, Julio C. Reyes $^{3}$, Ignacio A. Garcia-Godos ${ }^{1,4}$, \\ Karina Onton-Silva', Joanna Alfaro-Shigueto ${ }^{1,5}$ \\ ${ }^{1}$ Peruvian Centre for Cetacean Research (CEPEC), Museo de Delfines, Pucusana, Lima 20, Peru \\ ${ }^{2}$ Veterinary Sciences Division, Department of Agriculture and Rural Development, Stormont, Belfast BT4 3SD, UK \\ ${ }^{3}$ Areas Costeras y Recursos Marinos (ACOREMA), Av. San Martín 1471, Pisco, Peru \\ ${ }^{4}$ Area de Aves y Mamíferos Marinos, Instituto del Mar del Perú, Apdo. 22, Callao, Peru \\ ${ }^{5}$ Asociación ProDelphinus, Jiron Octavio Bernal 572-5, Lima 11, Peru \\ ${ }^{6}$ Present address: Centre for Cetacean Conservation Medicine, CEPEC, Waldspielplatz 11, 82319 Starnberg, Germany
}

\begin{abstract}
Miscellaneous lesions of the head, skull, teeth, trunk, appendages, skin and genital tract were observed in 120 of 930 long-beaked common dolphins Delphinus capensis taken in fisheries off Peru between 1985 and 2000. Seven subsamples were defined according to the varying field sampling protocols. Forty-two dolphins showed at least 2 types of injuries or diseases affecting 1 or more organs. The majority ( 5 of 7 ) of traumas encountered were diagnosed as caused by violent, fisheriesrelated interactions, and the skin in $20.4 \%$ of specimens $(\mathrm{n}=54)$ showed healed scars from such interactions. Prevalences of malformations and traumas of crania ( $\mathrm{n}=103$ ) were 2.9 and $1.9 \%$, respectively. Lytic cranial lesions were present in $31.1 \%$ of dolphins $(\mathrm{n}=103$ ) and accounted for $84.2 \%$ of all bone injuries. Skull damage diagnostic for Crassicauda sp. infestation was encountered in $26.5 \%$ of dolphins $(\mathrm{n}=98)$ and did not differ among sex and age classes. Crassicauda sp. and tooth infections were responsible for, respectively, 78.8 and $6.1 \%$ of the lytic lesions. Adult dolphins showed a high prevalence of worn and broken teeth $(35 \%, \mathrm{n}=20)$ as well as damaged alveoli $(20 \%, \mathrm{n}=70)$. Prevalence of 'paired teeth', a congenital condition, was $9.4 \%(\mathrm{n}=32)$. Lesions of the head, body and appendages were present in 10 dolphins and included traumas, deformations (e.g. scoliokyphosis and brachygnathia) and chronic mastitis. Ovarian cysts suggestive of follicular cysts were observed in 1 of 24 females. Chronic orchitis affected 1 of 78 males. Of 12 dolphins 2 had vesicular lesions of the penis. Prevalence of cutaneous lesions, abnormalities and scars ranged between $1.8 \%(\mathrm{n}=56)$ and $48.2 \%(\mathrm{n}=27)$.
\end{abstract}

KEY WORDS: Delphinus capensis · Diseases · Skull · Crassicauda sp. · Skin · Malformations · Fisheries interactions · Traumas $\cdot$ Ovarian cysts $\cdot$ Orchitis

\section{INTRODUCTION}

As part of a comprehensive study of the biology of common dolphins (genus Delphinus) in Pacific South America, we investigated the occurrence of diseases and lesions in various organs and tissues, as well as congenital malformations, in the long-beaked common dolphin D. capensis.

In the Southeast Pacific Ocean, Delphinus capensis is known to inhabit neritic waters characterised by strong upwelling off Peru and Chile, south to at least $28^{\circ} \mathrm{S}$ (Sanino et al. 2003a). This species is 1 of several 
delphinids that has suffered high levels of mortality in various types of fisheries in coastal Peru from at least about 1987 until the present (Van Waerebeek \& Reyes 1994, Van Waerebeek et al. 1997, 1999, 2002). In contrast, short-beaked common dolphins D. delphis are only occasionally landed. Their habitat off the coast of Peru is essentially oceanic, which puts them infrequently in the path of fisheries that operate on the continental shelf out of Peruvian ports, resulting in comparatively low mortality levels and very few specimens available for necropsy.

We previously documented infections by morbilliviruses, poxviruses and Brucella sp. in Delphinus capensis from coastal Peru (Van Bressem \& Van Waerebeek 1996, Van Bressem et al. 1998a, 2001a). Lesions of the skull as well as genital and lingual warts, possibly caused by papillomaviruses, were also reported on previously (Van Bressem et al. 1996, 2001b). This paper describes diseases, lesions, traumas and malformations of the skull, head, trunk and appendages as well as of the skin and genital tract. It aims to improve our knowledge of pathological factors that may influence modal health, net recruitment and, thus indirectly, population size, and to stimulate further research into possible implications for management. Published information on diseases in common dolphins worldwide is critically reviewed.

\section{MATERIALS AND METHODS}

Almost all study specimens of Delphinus capensis were captured by local fishermen in coastal waters off Peru in the period 1985 to 2000, either in artisanal drift gillnets, purse-seine nets set for small schooling fishes, or using harpoons. Specimens were collected or examined in a $1050 \mathrm{~km}$ coastal strip, stretching from Playa Chucho, Paracas Reserve $\left(13^{\circ} 48^{\prime} \mathrm{S}, 76^{\circ} 24^{\prime} \mathrm{W}\right)$, in south central Peru, to Parachique $\left(05^{\circ} 34^{\prime} \mathrm{S}, 80^{\circ} 52^{\prime} \mathrm{W}\right)$, northern Peru. Biological and pathological data and samples were either collected in situ at the wharves where the dolphins were landed or while beach-combing. A total of 930 individuals were examined, including 2 critically injured females landed alive and euthanized, 867 fresh carcasses (most less than $24 \mathrm{~h}$ post-mortem, condition 2; see Geraci \& Lounsbury 1993) and miscellaneous remains (condition 3 to 5) of another 61 individuals. Seven main subsamples (A-G) were defined, due to the unpredictable availability of carcasses and organs, variable field conditions and differing research priorities of the participating scientists. Population biologists studied any aberrant morphology so as to exclude individual or population-linked variation, before it was classified as a congenital malformation or other pathology.
Dolphin specimens. Maturity, an important factor in epidemiological considerations, was recorded as accurately as possible. Dolphins were considered cranially mature when advanced fusion was present in the frontal-supraoccipital suture or in at least 2 of 6 other indicative cranial sutures (Van Waerebeek 1992, 1993).

Females were classified as sexually mature if the ovaries showed at least 1 corpus luteum (CL) or corpus albicans (CA), or if lactation or pregnancy (visible foetus) was evident (Van Waerebeek et al. 1994). Field results were validated in the laboratory for 17 females by transverse sectioning of ovaries according to standard techniques (e.g. Perrin et al. 1976). The sexual maturity of 59 males was examined by histology of testes and epididymides (Hohn et al. 1985). In the absence of histological results, males were considered sexually mature if seminal fluid was detected in at least one freshly cut epididymis during macroscopical examination in the field. When sexual maturity status could not be determined directly, it was inferred based on a preliminary approximation for the mean standard body length (SL) at sexual maturation for this population, i.e. males below $215 \mathrm{~cm}$ and females below $200 \mathrm{~cm}$ were assumed immature, while males above $220 \mathrm{~cm}$ and females above $210 \mathrm{~cm}$ were considered mature (CEPEC unpubl. data). Females and males measuring 200 to 210 and 215 to $220 \mathrm{~cm}$, respectively, were classified as of unknown sexual maturity.

Crania and teeth. The crania of 103 specimens (73 complete skulls, 30 calvariae) of long-beaked common dolphins landed or washed ashore dead, on the Peruvian coast in 1986 to 2000 (Table 1), were examined for the presence of bone lesions and abnormalities, including the typical, apparently irreversible basket-like lesions caused by the nematodes Crassicauda spp. (e.g. Raga et al. 1982, Dailey 1985). Skulls are deposited at the Museo de Delfines (Pucusana) and the research collection of 'Areas Costeras y Recursos Marinos' (ACOREMA, Pisco), in Peru.

Teeth and tooth alveoli were checked for lesions in 2 samples of 23 and 103 dolphins, respectively (Table 1). The presence of 'paired teeth' (a congenital malformation, see below) was also studied in 10 fresh dolphins (Table 1) caught off Cerro Azul in 1993, and 1 skull (MFB-221) of this sample was collected.

Head, trunk and appendages. The complete, fresh carcasses of 859 long-beaked common dolphins were examined by at least one of the authors between 1985 and 1994. The prevalence of lesions, malformations and abnormalities was estimated separately for 2 subsamples, depending on the researchers, sampling periods and the ports where the animals were landed.

Subsample A: External features, including lesions and abnormalities, were examined by K.V.W. and J.C.R. in 314 dolphins (Table 1) taken off central 
Table 1. Delphinus capensis. Composition of samples and subsamples from Peru examined for lesions and diseases from 1985 to 2000. The terms 'Immature', 'Mature' and 'Unknown' refer to cranial maturity for the 'Crania and teeth' sample unless otherwise indicated, and to sexual maturity for all other samples. N: number

\begin{tabular}{|c|c|c|c|c|c|c|c|c|c|c|c|c|c|}
\hline & \multirow{2}{*}{$\begin{array}{c}\text { Total } \\
\mathrm{N}\end{array}$} & \multicolumn{4}{|c|}{ Males } & \multicolumn{4}{|c|}{$\longrightarrow$ Females } & \multicolumn{4}{|c|}{ _ Undetermined sex } \\
\hline & & $\mathrm{N}$ & Immature & Mature & Unknown & $\mathrm{N}$ & Immature & Mature & Unknown & $\mathrm{N}$ & Immature & Mature & Unknown \\
\hline \multicolumn{14}{|l|}{ Crania and teeth } \\
\hline Crania & 103 & 33 & 2 & 31 & 0 & 11 & 2 & 9 & 0 & 59 & 18 & 28 & 13 \\
\hline Tooth alveoli & 103 & 34 & 2 & 32 & 0 & 11 & 2 & 9 & 0 & 58 & 18 & 29 & 11 \\
\hline Teeth (skull) & 23 & 10 & 0 & 10 & 0 & 3 & 0 & 3 & 0 & 10 & 1 & 7 & 2 \\
\hline Teeth (fresh carcass) ${ }^{a}$ & 10 & 9 & 8 & 1 & 0 & 1 & 0 & 0 & 1 & 0 & 0 & 0 & 0 \\
\hline \multicolumn{14}{|c|}{ Head, trunk and appendages } \\
\hline Subsample A & 314 & 179 & 144 & 19 & 16 & 135 & 82 & 20 & 33 & 0 & 0 & 0 & 0 \\
\hline Subsample B & 545 & 355 & 267 & 64 & 24 & 190 & 127 & 34 & 29 & 0 & 0 & 0 & 0 \\
\hline \multicolumn{14}{|l|}{ Skin } \\
\hline Subsample C & 314 & 179 & 144 & 19 & 16 & 135 & 82 & 20 & 33 & 0 & 0 & 0 & 0 \\
\hline Subsample D & 27 & 16 & 16 & 0 & 0 & 11 & 11 & 0 & 0 & 0 & 0 & 0 & 0 \\
\hline Subsample E & 56 & 44 & 34 & 10 & 0 & 12 & 10 & 0 & 2 & 0 & 0 & 0 & 0 \\
\hline \multicolumn{14}{|l|}{ Genital tract } \\
\hline Subsample F & 102 & 78 & 42 & 35 & 1 & 24 & 10 & 14 & 0 & 0 & 0 & 0 & 0 \\
\hline Subsample G & 126 & 61 & 45 & 16 & 0 & 65 & 51 & 14 & 0 & 0 & 0 & 0 & 0 \\
\hline
\end{tabular}

Peru and Chimbote $\left(09^{\circ} 05^{\prime} \mathrm{S}, 78^{\circ} 36^{\prime} \mathrm{W}_{;} 1\right.$ individual $)$ between 1985 and 1989.

Subsample B: External features of 545 dolphins (Table 1) captured off central and northern Peru from 1990 to 1994 were examined by several CEPEC scientists. Greater attention was paid to anomalies and injuries in this period than in the 1985 to 1989 period.

Skin. Although dolphins were examined for injuries since 1985, cutaneous marks were not recorded systematically until late 1989. From 1991 onwards we routinely investigated skin lesions (excluding tooth rakes) in Delphinus capensis. Three subsamples were defined according to the research focus, the sampling period and the port of landing.

Subsample C: The external features of 314 dolphins (Table 1) caught off central Peru (all but 1 from Chimbote) in 1985 to 1989 were examined by K.V.W. and J.C.R.. Skin marks were reported opportunistically.

Subsample D: Twenty-seven freshly dead dolphins (Table 1) landed at Ancon (11 $\left.47^{\prime} \mathrm{S}, 7^{\circ} 11^{\prime} \mathrm{W}\right)$ in 1991 and 1992, were visually checked by A.G.G. for the presence of skin injuries. However, natural history and port monitoring, not skin diseases, were the research priorities and few lesions were photographed. A bias towards positive specimens may have occurred and prevalence of lesions is probably overestimated.

Subsample E: The integument of a random sample of 56 dolphins (Table 1), taken off central Peru and northern Peru ( $=2$ ) in 1993 and 1994, was examined for tattoos (irregular, grey, black or yellowish, stippled skin lesions caused by poxviruses; Van Bressem \& Van Waerebeek 1996) and other skin marks by several of the authors of the current study (M.F.B., K.V.W., K.O.S., A.G.G.). This unbiased sample provides reliable prevalence data.

\section{Genital tract.}

Subsample F: The ovaries of 24 females and the testes of 78 males were checked in the field for the presence of lesions and abnormalities (Table 1). Of these, the gonads of 18 females and 60 males were also studied by histology. The penises of 12 dolphins (3 mature, 9 immature) were inspected for genital warts (Van Bressem et al. 1996) and other lesions. All animals were landed at ports of central and northern Peru between 1987 and 1994.

Subsample G: Data on genital tract lesions were gleaned from standardised specimen datasheets and miscellaneous field notes archived at the CEPEC field station. They related to 65 females and 61 males (Table 1) taken off central Peru between 1985 and 1992.

Laboratory protocols. Representative tissue samples were fixed in a $10 \%$ buffered formaldehyde solution, dehydrated in a graded series of ethanol and embedded in paraffin wax. Tissue sections $(5 \mu \mathrm{m})$ were stained with haematoxylin and eosin (H\&E). Ultrathin sections of skin lesions from 5 long-beaked common dolphins (Subsample D) were screened for virus particles by transmission electron microscopy (TEM), following methodology described in Van Bressem et al. (1993a).

Prevalence and statistical testing. We examined whether the prevalence of lesions varied with sex and sexual or cranial maturity (as a proxy for age). To determine potential age-relatedness of skull injuries and malformations, we divided the sample into cra- 
nially mature and immature individuals. Where the alterations observed could not with certainty be classified as pathological, the relevant specimens were excluded from statistical tests; hence, the differences in some sample sizes. Prevalence refers to the amount of lesions and disease in samples and subsamples at the time of examination, without distinction between old and new cases (Thrusfield 1986). Significance of differences in prevalence $(\alpha=0.05)$ was verified with chi-square contingency tests or 1-tailed Fisher's exact tests (Swinscow 1981).

\section{RESULTS}

A review of earlier studies of diseases in Delphinus spp. worldwide is compiled in Table 2. In the present study, miscellaneous lesions of the skull, teeth, head, trunk, appendages, skin and genital tract were observed in 120 out of 930 dolphins. Forty-two specimens had at least 2 types of lesions that affected 1 or more organs/tissues. When pooled with previously published data on lingual and genital warts (Van Bressem et al. 1996), this number increased to 48 (Table 3). A large, fully mature male (KVW-2403) had injuries in at least 6 different organs (Table 3). Violent interactions with fishing gear or human actions, including clubbing, were considered the cause of severe traumas observed in the skull, on the head trunk and appendages in 5 of 7 dolphins (see below).

\section{Lesions of the skull}

Crassicauda-caused cranial lesions. Round, lytic bone lesions with a basket-like appearance, typically associated with Crassicauda spp. infestation, were found in the cranial sinuses of 18 adults, 5 immatures and 3 dolphins of unknown maturity (Table 4, Fig. 1). The pterygoid bones were affected in $96 \%$ of the 26 positive dolphins. The frontal, alisphenoid, palatine, maxillary and exoccipital bones were occasionally altered. Crassicauda injuries were extensive in 3 of 5 immature and in 4 of 18 infested adults.

Osteomyelitis. Acute or chronic bone infection characterised by bone destruction and new bone formation was observed in 2 mature dolphins (Table 4). In 1 skull (KVW-2401) ca. $50 \mathrm{~mm}$ of irregular new bone, including 2 thorn-like protuberances and a $7 \times 7 \mathrm{~mm}$ fenestration, was visible on the left pterygoid and palatine. Further, bone lysis deformed the outer edge of the left maxillary for over $55 \mathrm{~mm}$ at the base of the rostrum.

Another skull (KVW-2400) showed a large area (35 $\times$ $24 \mathrm{~mm}$ ) of bone destruction on the latero-dorsal side of the left mandible, behind the tooth row (Fig. 2). The bone lining the area had a rough aspect with several longitudinal depressions, each about $4 \mathrm{~mm}$ deep.

Osteolysis. Cranial bone lysis that did not seem related to Crassicauda nematode infestation was detected in 5 dolphins (Table 4).

In a cranially mature male (JAS-17), a channel-like fistula vertically traversed the left maxillary, dorsally from tooth alveolus \#16 to open into the buccal cavity (Fig. 3a). Adjacent lytic lesions on the buccal side of both maxillaries and on the right premaxillary were likely the continuation of the fistula (Fig. 3). Several alveoli on the left maxillary were occluded and, at 2 sites, interalveolar septa were replaced by spongiform bony tissue. The fistula and other lytic lesions likely originated from caries and spreading infection. Similarly, in another mature male (RBC-19), a small channel $(4 \times 5 \mathrm{~mm})$ perforated the left maxillary buccal dorsally at alveolus \#33, probably also the result of tooth infection.

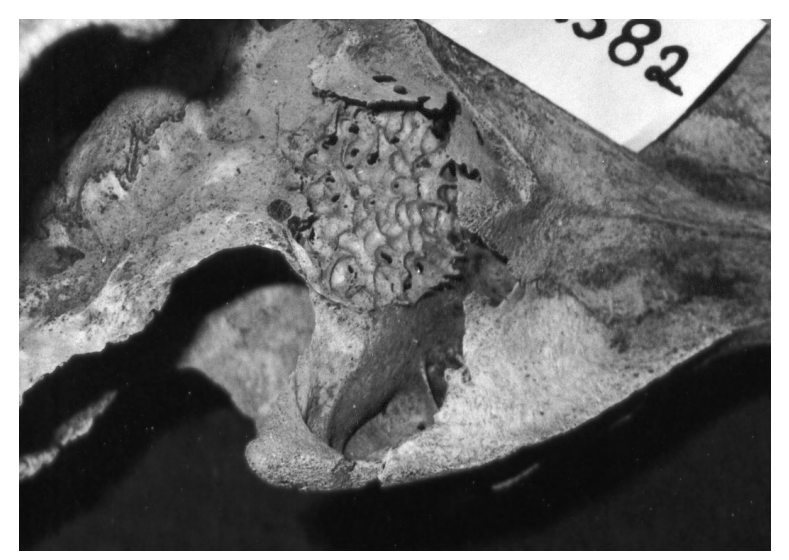

Fig. 1. Delphinus capensis. Crassicauda lesions in the right pterygoid of a cranially adult dolphin (KVW-2382)

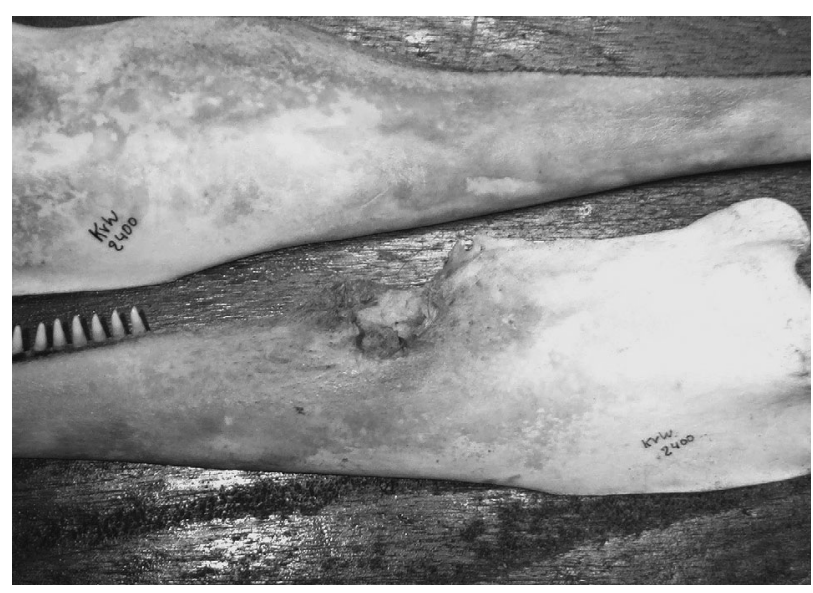

Fig. 2. Delphinus capensis. Large area of osteomyelitis on the latero-dorsal side of the left mandible of a mature dolphin (KVW-2400) 
Table 2. Delphinus delphis and D. capensis. Worldwide review of diseases and lesions. NE $=$ northeast, $\mathrm{SE}=\mathrm{southeast}, \mathrm{NW}=$ northwest, UK = United Kingdom

\begin{tabular}{|c|c|c|c|}
\hline Species & Type of disease & Ocean province & Source \\
\hline & Organic diseases (excluding bones) & & \\
\hline D. delphis ponticus & Fibroma on surface of right testis & Black Sea & Birkun et al. (1999) \\
\hline D. delphis & Multicentric cholangiocarcinoma & Coasts of UK & Baker (1992) \\
\hline D. delphis & Cystic pancreas & Coasts of UK & Baker (1992) \\
\hline D. delphis & Interstitial nephritis & Coasts of UK & Baker (1992) \\
\hline D. delphis & Hydrocephalus & Coasts of UK & Baker (1992) \\
\hline D. delphis & Vaginal calculi & NE Atlantic, coasts of UK, NE Pacific & $\begin{array}{l}\text { Sawyer \& Walker (1977), Baker } \\
\text { (1992), Lopez \& Benavente (1993) }\end{array}$ \\
\hline D. delphis & Gastric leiomyoma & NE Pacific & Cowan et al. (1986) \\
\hline D. delphis & Cardiac lesions & NE Pacific & Cowan et al. (1986) \\
\hline D. delphis & Arteriosclerosis & NE Pacific & Cowan et al. (1986) \\
\hline D. delphis & Vaginal mass & NE Pacific & Benirschke et al. (1984) \\
\hline D. delphis & $\begin{array}{l}\text { Epididymal abcess associated with } \\
\text { Monorygma sp. }\end{array}$ & NE Pacific & Cowan et al. (1986) \\
\hline D. delphis & Leydig cell tumour (testes) & NE Pacific & Cowan et al. (1986) \\
\hline D. delphis & Gastric ulceration & NE Pacific, coasts of UK & Cowan et al. (1986), Baker (1992) \\
\hline D. delphis & Hepatitis & Port Philip Bay, Australia (Indian Ocean) & Dixon (1984) \\
\hline \multirow[t]{2}{*}{ D. delphis } & Bacterial pneumonia & SE Pacific & Sanino et al. (2003b) \\
\hline & Lesions of the bones & & \\
\hline D. delphis & $\begin{array}{l}\text { Malformations of the cranium, } \\
\text { traumatic lesions of vertebrae }\end{array}$ & Port Philip Bay, Australia (Indian Ocean) & Dixon (1984) \\
\hline D. delphis & Fibrous osteodistrophy & NE Pacific & Flom et al. (1978) \\
\hline D. capensis & $\begin{array}{l}\text { Crassicauda sp. lesions in } \\
\text { pterygoids, frontals, alisphenoids, } \\
\text { palatines, maxillaries and exoccipitals }\end{array}$ & SE Pacific & $\begin{array}{l}\text { Van Bressem et al. (2001b), } \\
\text { Montes-Iturrizaga (2003), } \\
\text { present study }\end{array}$ \\
\hline D. capensis & $\begin{array}{l}\text { Osteomyelitis in pterygoid, palatine, } \\
\text { mandible }\end{array}$ & SE Pacific & $\begin{array}{l}\text { Van Bressem et al. (2001b), } \\
\text { Montes-Iturrizaga (2003), } \\
\text { present study }\end{array}$ \\
\hline D. capensis & $\begin{array}{l}\text { Osteolysis of the maxillaries and } \\
\text { premaxillaries }\end{array}$ & SE Pacific & $\begin{array}{l}\text { Van Bressem et al. (2001b), } \\
\text { Montes-Iturrizaga (2003), } \\
\text { present study }\end{array}$ \\
\hline D. capensis, D. delphis & $\begin{array}{l}\text { Traumas (healed fractures and } \\
\text { perforations) }\end{array}$ & SE Pacific & $\begin{array}{l}\text { Van Bressem et al. (2001b), } \\
\text { Montes-Iturrizaga (2003), } \\
\text { Sanino et al. (2003b); present study }\end{array}$ \\
\hline \multirow[t]{2}{*}{ D. capensis } & $\begin{array}{l}\text { Congenital and acquired mal- } \\
\text { formations (maxillaries, premaxillaries } \\
\text { and mandibles) }\end{array}$ & SE Pacific & $\begin{array}{l}\text { Van Bressem et al. (2001b), } \\
\text { Montes-Iturrizaga (2003), } \\
\text { present study }\end{array}$ \\
\hline & Dental and periodontal diseases & & \\
\hline D. capensis, D. delphis & Broken, worn and missing teeth & Coasts of the UK, SE Pacific & $\begin{array}{l}\text { Baker (1992), Montes-Iturrizaga } \\
\text { (2003), present study }\end{array}$ \\
\hline D. capensis & Paired teeth & SE Pacific & Present study \\
\hline \multirow[t]{2}{*}{ D. capensis } & Occluded alveoli & SE Pacific & $\begin{array}{l}\text { Montes-Iturrizaga (2003), } \\
\text { present study }\end{array}$ \\
\hline & Infectious diseases & & \\
\hline D. capensis, D. delphis & Morbillivirus infection & $\begin{array}{l}\text { SW Indian Ocean, East Pacific, } \\
\text { NW Atlantic, NE Atlantic, Mediterranean } \\
\text { Sea, coasts of NW Europe }\end{array}$ & $\begin{array}{l}\text { Duignan et al. (1995), Reidarson } \\
\text { et al. (1998), Van Bressem et al. } \\
\text { (1993b, 1998a,b, 2001c), Visser } \\
\text { et al. (1993) }\end{array}$ \\
\hline D. delphis ponticus & Morbillivirus infection & Black Sea & Birkun et al. (1999) \\
\hline D. capensis & Tattoo skin disease (poxvirus) & SE Pacific & $\begin{array}{l}\text { Van Bressem \& Van Waerebeek } \\
\text { (1996) }\end{array}$ \\
\hline D. capensis & Genital warts (possibly papillomavirus) & SE Pacific & Van Bressem et al. (1996) \\
\hline D. capensis, D. delphis & Brucella sp. infection & SE Pacific, NE Atlantic, North Sea & $\begin{array}{l}\text { Ross et al. (1996), Jepson et al. } \\
\text { (1997), Van Bressem et al. (2001a) }\end{array}$ \\
\hline D. delphis & $\begin{array}{l}\text { Dolphin rhabdovirus-like virus (DRV) } \\
\text { infection }\end{array}$ & $\begin{array}{l}\text { Likely coasts of NW Europe } \\
\text { (specific origin not given) }\end{array}$ & Osterhaus et al. (1993) \\
\hline Delphinus spp. & Pneumonia & NE Pacific, coasts of UK & Cowan et al. (1986), Baker (1992) \\
\hline Delphinus spp. & Bacterial enteritis & Unknown (captive individual) & Sweeney \& Ridgway (1975) \\
\hline
\end{tabular}


Table 3. Delphinus capensis. Multiple lesions in dolphins from Peruvian waters. Specimens $(\mathrm{n}=48)$ are ordered by standard body length (SL). Initials AGG, JAS, JCR, KOS, KVW and MFB refer to authors of the present study. RBC = Ruth Bello Calvo, $\mathrm{ACO}=$ Areas Costreas y Recursos Marinos, $\mathrm{imm}=$ immature, mat $=$ mature, indet $=$ indeterminate. Parentheses indicate that sexual maturity was inferred from SL

\begin{tabular}{|c|c|c|c|c|c|c|c|c|}
\hline Specimen & $\begin{array}{l}\text { Date } \\
(\mathrm{d} / \mathrm{mo} / \mathrm{yr})\end{array}$ & $\begin{array}{l}\text { Locality } \\
\text { (collected) }\end{array}$ & Sex & $\begin{array}{l}\mathrm{SL} \\
(\mathrm{cm})\end{array}$ & $\begin{array}{l}\text { Cranial } \\
\text { maturity }\end{array}$ & $\begin{array}{l}\text { Sexual } \\
\text { maturity }\end{array}$ & $\begin{array}{l}\text { Organs } \\
\text { affected }\end{array}$ & Lesions \\
\hline AGG-591 & $18 / 2 / 92$ & Ancon & $\mathrm{F}$ & 152.5 & indet & $\mathrm{imm}$ & Skin & Tattoos, punctiform marks \\
\hline AGG-405 & $5 / 9 / 91$ & Ancon & $\mathrm{F}$ & 167.5 & indet & $\mathrm{imm}$ & Body, skin & $\begin{array}{l}\text { Chronic fibriotic reaction } \\
\text { on tailstock, kyphosis, tattoos }\end{array}$ \\
\hline AGG-603 & $25 / 2 / 92$ & Ancon & $\mathrm{F}$ & 171 & indet & imm & Body, skin & $\begin{array}{l}\text { Punctiform marks on the whole } \\
\text { body, very thin animal }\end{array}$ \\
\hline KOS-123 & 19/6/93 & Cerro Azul & F & 174.5 & indet & (imm) & Skin & Tattoos, 3 scars on tailstock \\
\hline KVW-522 & $11 / 1 / 87$ & Pucusana & $\mathrm{F}$ & 184.5 & indet & $\mathrm{imm}$ & Head, skin & $\begin{array}{l}\text { Scar behind left eye, broken and } \\
\text { healed mandibular ramus }\end{array}$ \\
\hline MFB-228 & 6/6/93 & Cerro Azul & M & 184.5 & indet & (imm) & Skin & Tattoo, 2 scars on head and left flank \\
\hline AGG-575 & $17 / 11 / 91$ & Ancon & $\mathrm{F}$ & 186 & indet & $\mathrm{imm}$ & Skin & $\begin{array}{l}\text { Tattoos, punctiform marks on the whole } \\
\text { body }\end{array}$ \\
\hline MFB-232 & 6/6/93 & Cerro Azul & $\mathrm{M}$ & 188.5 & indet & (imm) & Skin & Tattoos, scar on head \\
\hline AGG-592 & $18 / 2 / 92$ & Ancon & M & 191.5 & indet & $\mathrm{imm}$ & Skin & $\begin{array}{l}\text { Tattoos, punctiform marks on the whole } \\
\text { body }\end{array}$ \\
\hline MFB-219 & $15 / 5 / 93$ & Cerro Azul & M & 192 & indet & imm & $\begin{array}{l}\text { Teeth, skin, } \\
\text { genital slit }\end{array}$ & $\begin{array}{l}\text { Tattoos, scar on head, genital papilloma, } \\
\text { paired teeth }\end{array}$ \\
\hline MFB-297 & 21/8/93 & Cerro Azul & $\mathrm{F}$ & 192 & indet & (imm) & Skin & Tattoos, large scar on tailstock \\
\hline MFB-226 & 4/6/93 & Cerro Azul & M & $>194$ & indet & (imm) & Skin & $\begin{array}{l}\text { Tattoos, dark circles on the belly and } \\
\text { right flank }\end{array}$ \\
\hline AGG-735 & $27 / 2 / 93$ & Culebras & $\mathrm{F}$ & 196 & indet & (imm) & Skin & Tattoos, punctiform marks on the head \\
\hline MFB-264 & 8/8/93 & Cerro Azul & M & 197 & indet & (imm) & Skin & Tattoos, scar on tailstock \\
\hline MFB-265 & 8/8/93 & Cerro Azul & M & 197 & indet & (imm) & Skin & Tattoo, scar on the right flank \\
\hline MFB-258 & $8 / 8 / 93$ & Cerro Azul & M & 197.5 & indet & (imm) & Skin, genitals & Tattoos, scar on head, genital papilloma \\
\hline AGG-576 & 17/11/91 & Ancon & $\mathrm{F}$ & 198 & indet & (imm) & Skin & $\begin{array}{l}\text { Remains of tattoos, punctiform marks on } \\
\text { the whole body }\end{array}$ \\
\hline KOS-94 & 2/6/93 & Cerro Azul & M & 198 & indet & $\mathrm{imm}$ & Skin & Tattoos, scar \\
\hline AGG-606 & $1 / 3 / 92$ & Ancon & M & 199.5 & indet & imm & Skin & $\begin{array}{l}\text { Tattoos, punctiform marks all over the } \\
\text { body }\end{array}$ \\
\hline MFB-269 & 8/8/93 & Cerro Azul & M & 199.5 & indet & (imm) & Skin & Tattoos, punctiform marks on belly \\
\hline MFB-312 & $27 / 10 / 93$ & Cerro Azul & M & 200 & indet & (imm) & Skin & $\begin{array}{l}\text { Tattoos, dark circles, scars on head } \\
\text { and right flipper }\end{array}$ \\
\hline MFB-675 & $9 / 7 / 94$ & Cerro Azul & M & 200.5 & indet & imm & Skin, penis & $\begin{array}{l}\text { Tattoos, vesicular lesion on penis, } \\
\text { anomalous skin pigmentation of } \\
\text { left flipper }\end{array}$ \\
\hline MFB-86 & 26/3/93 & Cerro Azul & M & 200.5 & indet & imm & Skin & $\begin{array}{l}\text { Tattoos, coronet marks on the belly, } \\
\text { scar on left flipper }\end{array}$ \\
\hline MFB-220 & $15 / 5 / 93$ & Cerro Azul & $\mathrm{F}$ & 202 & indet & indet & $\begin{array}{l}\text { Teeth, beak, } \\
\text { genital slit }\end{array}$ & $\begin{array}{l}\text { Brachygnathia, paired teeth, genital } \\
\text { papilloma }\end{array}$ \\
\hline MFB-281 & $12 / 8 / 93$ & Cerro Azul & $\mathrm{M}$ & 204 & indet & (imm) & Skin & Tattoos, scar on flipper \\
\hline MFB-510 & $18 / 5 / 94$ & Cerro Azul & M & 207 & indet & $\mathrm{imm}$ & Penis, skin & $\begin{array}{l}\text { Tattoos, round skin marks on flanks and } \\
\text { belly, vesicular lesions on penis }\end{array}$ \\
\hline MFB-218 & $15 / 5 / 93$ & Cerro Azul & M & 209 & indet & $\mathrm{imm}$ & Skin & Tattoos, scar on head \\
\hline MFB-87 & 26/3/93 & Cerro Azul & $\mathrm{F}$ & 210 & indet & indet & Skin & Tattoos, 1 scar on right flank \\
\hline MFB-508 & $17 / 5 / 94$ & Cerro Azul & M & 210.5 & indet & $\mathrm{imm}$ & Skin & Tattoos, round skin marks on belly \\
\hline MFB-191 & $13 / 5 / 93$ & Cerro Azul & $\mathrm{F}$ & 211.5 & mat & mat & Skull, ovary & Crest on rostrum, multicystic left ovary \\
\hline MFB-259 & 8/8/93 & Cerro Azul & M & 214 & indet & $\mathrm{imm}$ & Skin & Tattoos, dark circles on belly \\
\hline MFB-230 & 6/6/93 & Cerro Azul & M & 221 & indet & (mat) & Skin, genitals & Dark circles, scar, genital papillomas \\
\hline MFB-229 & 6/6/93 & Cerro Azul & M & 224.5 & mat & mat & Skin, body & $\begin{array}{l}\text { Tattoos, dark circles on left flank and } \\
\text { belly, emaciated }\end{array}$ \\
\hline MFB-142 & $15 / 4 / 93$ & Cerro Azul & M & 226.5 & mat & mat & $\begin{array}{l}\text { Skull, alveoli, } \\
\text { skin }\end{array}$ & $\begin{array}{l}\text { Tattoos, } 1 \text { alveolus closed, extensive } \\
\text { Crassicauda sp. lesions in left pterygoid }\end{array}$ \\
\hline KVW-2404 & $31 / 5 / 94$ & Pucusana & M & 228.5 & mat & mat & $\begin{array}{l}\text { Teeth, skin, } \\
\text { tongue }\end{array}$ & $\begin{array}{l}\text { Broken teeth, round marks on belly, } \\
\text { lingual warts }\end{array}$ \\
\hline RBC-21 & $26 / 3 / 93$ & Chimbote & M & 234 & mat & mat & $\begin{array}{l}\text { Skull, alveoli, } \\
\text { flipper }\end{array}$ & $\begin{array}{l}\text { Insertion of flipper broken, lesions of } \\
\text { alveoli, congenital malformation of } \\
\text { the beak }\end{array}$ \\
\hline AGG-761 & $12 / 8 / 93$ & Chimbote & M & 236.5 & mat & mat & Skin & Tattoos, punctiform marks on the back \\
\hline KOS-90 & 1/6/93 & Cerro Azul & $\mathrm{F}$ & 239.5 & mat & mat & Skull & Lesions of the alveoli, broken teeth \\
\hline RBC-17 & $26 / 3 / 93$ & Chimbote & M & 240 & mat & mat & Skull, alveoli & $\begin{array}{l}\text { Lesions of the alveoli, Crassicauda sp. } \\
\text { lesions in right pterygoid }\end{array}$ \\
\hline
\end{tabular}


Table 3. (continued)

\begin{tabular}{|c|c|c|c|c|c|c|c|c|}
\hline Specimen & $\begin{array}{c}\text { Date } \\
(\mathrm{d} / \mathrm{mo} / \mathrm{yr})\end{array}$ & $\begin{array}{l}\text { Locality } \\
\text { (collected) }\end{array}$ & Sex & $\begin{array}{l}\mathrm{SL} \\
(\mathrm{cm})\end{array}$ & $\begin{array}{l}\text { Cranial } \\
\text { maturity }\end{array}$ & $\begin{array}{l}\text { Sexual } \\
\text { maturity }\end{array}$ & $\begin{array}{l}\text { Organs } \\
\text { affected }\end{array}$ & Lesions \\
\hline MFB-529 & $22 / 5 / 94$ & Cerro Azul & M & 240.5 & mat & mat & Teeth, genitals & Paired teeth, genital papilloma \\
\hline KVW-2403 & $31 / 5 / 94$ & Pucusana & M & 241.0 & mat & mat & $\begin{array}{l}\text { Teeth, alveoli, } \\
\text { skull, body, } \\
\text { skin, testis }\end{array}$ & $\begin{array}{l}\text { Lump on tailstock, orchitis, round marks } \\
\text { on the belly, Crassicauda sp. lesions } \\
\text { in right pterygoid, broken teeth, lesions } \\
\text { of alveoli }\end{array}$ \\
\hline RBC-19 & 26/3/93 & Chimbote & M & 241.5 & mat & mat & Skull, alveoli & Lesions of the alveoli, lysis in left maxillary \\
\hline ACO-63 & 1/8/98 & Paracas & M & 245 & mat & (mat) & Alveoli, teeth & Lesions of the alveoli and teeth \\
\hline JAS-17 & $24 / 6 / 93$ & Pucusana & M & 247.5 & mat & mat & Skull & $\begin{array}{l}\text { Lesions of the alveoli, lysis of maxillaries } \\
\text { and right premaxillary }\end{array}$ \\
\hline MFB-149 & 17/4/93 & Cerro Azul & M & 252 & mat & mat & Alveoli, teeth & Lesions of alveoli, broken teeth \\
\hline KVW-2400 & $25 / 10 / 93$ & Chancay & indet & indet & mat & indet & Skull, teeth & $\begin{array}{l}\text { Osteomyelitis of left mandible, } \\
\text { broken teeth }\end{array}$ \\
\hline MFB-174 & $25 / 4 / 93$ & Cerro Azul & indet & indet & mat & indet & Skull, teeth & Osteolysis of left maxillary, 1 broken tooth \\
\hline MFB-741 & $13 / 1 / 95$ & Matacaballo & indet & indet & mat & indet & Skull, alveoli & $\begin{array}{l}\text { Healed fracture of left mandible, } \\
\text { Crassicauda sp. lesions, lesions of alveoli }\end{array}$ \\
\hline
\end{tabular}

In a mature dolphin (AGG-621), a $20 \times 10 \mathrm{~mm}$ lytic lesion of the right maxillary communicated ventrally with the palatine sinus. A Crassicauda sp. aetiology was excluded as the lesion lacked the diagnostic basket-like osseous morphology.

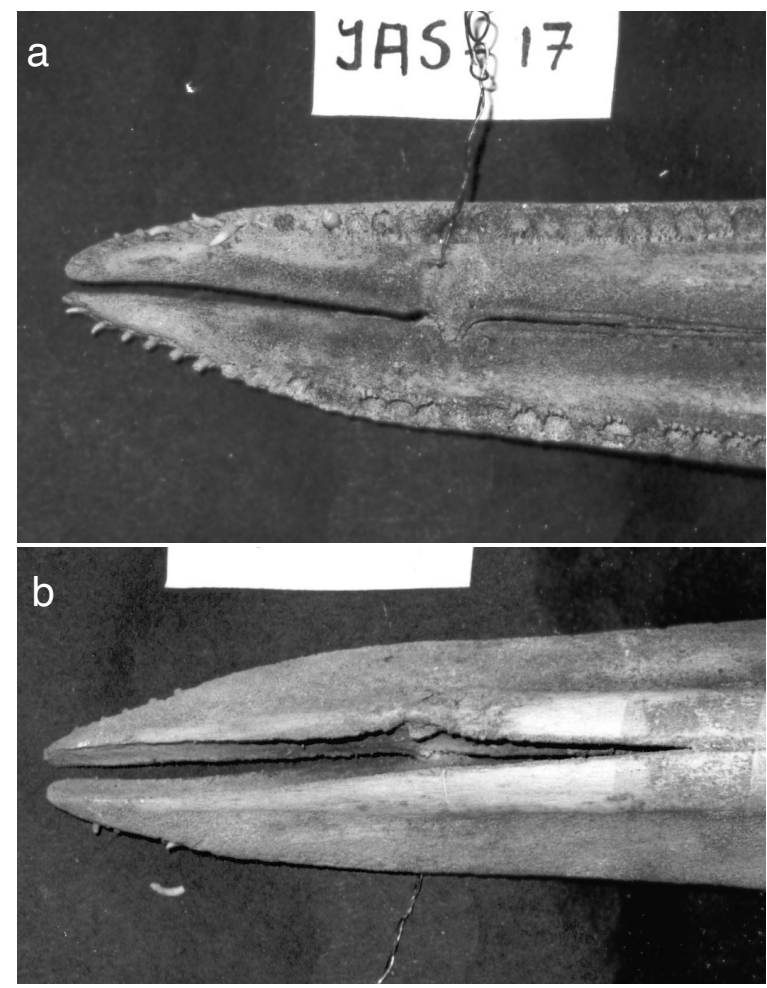

Fig. 3. Delphinus capensis. (a) Channel-like fistula traversing vertically the left maxillary and adjacent lytic lesions on the ventral side of both maxillaries and (b) on the right premaxillary are seen in cranially mature male JAS-17
In another mature dolphin (MFB-174), the left maxillary under the palatine keel was eroded over $24 \mathrm{~mm}$ and presented a perforating fistula lined by irregular bony tissue of unknown origin, possibly due to, or exacerbated by, a bacterial infection (Table 4, Fig. 4). A small, $8 \mathrm{~mm}$ wide fistula was located $60 \mathrm{~mm}$ from the neurocranium (Fig. 4). In a cranially immature dolphin (MFB-756) the distal extremities of the premaxillaries were partially dissolved and slightly deformed over a length of $71 \mathrm{~mm}$ (Fig. 5), but the aetiology here was unknown.

Congenital and acquired malformation. In JCR-1351, a cranially mature female dolphin, the distal half of the rostrum and lower jaw was curved upwards, its extremity forming an angle of approximately $45^{\circ}$ relative to the normal rostrum axis (Fig. 6). Moreover, the mandible

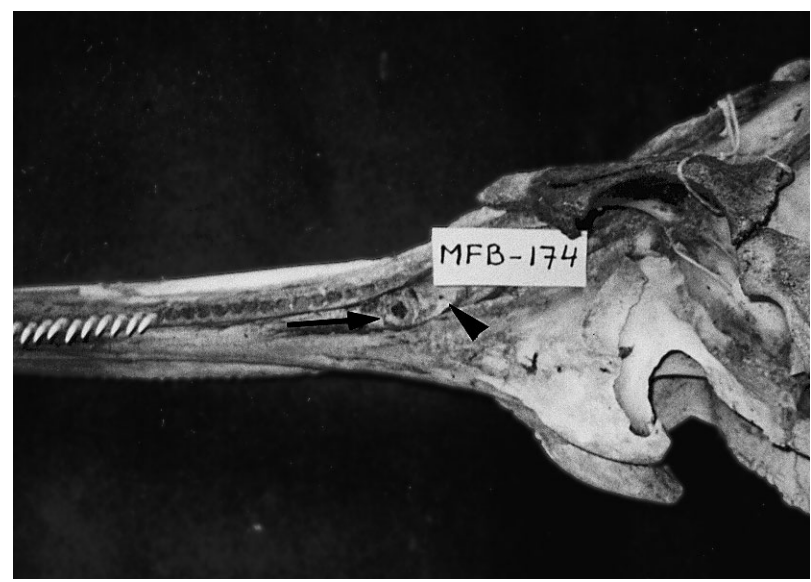

Fig. 4. Delphinus capensis. Area of bone erosion and perforating fistula (arrow) on the ventral side of the left maxillary, under the palatine keel in a mature dolphin of unknown sex (MFB-174). A small, $8 \mathrm{~mm}$ wide fistula is seen $60 \mathrm{~mm}$ closer to the neurocranium (arrowhead) 
Table 4. Delphinus capensis. Lesions of the skull, head, trunk, appendages and genital tract found in 46 dolphins from Peruvian waters. Initials AGG, JAS, JCR, KVW and MFB refer to authors of the present study. RBC= Ruth Bello Calvo, LAS = Luis A. Santillan, MWC = Mark W. Chandler, ACO = Areas Costeras y Recursos Marinos. SL = standard body length, imm $=$ immature, mat $=$ mature, pub $=$ pubescent, indet $=$ indeterminate. Parentheses indicate that sexual maturity was inferred from SL; -: precise day of collection unknown

\begin{tabular}{|c|c|c|c|c|c|c|c|}
\hline Organs/ tissues affected & Specimen & $\begin{array}{c}\text { Date } \\
\text { (d/mo/yr) }\end{array}$ & $\begin{array}{l}\text { Locality } \\
\text { (collected) }\end{array}$ & Sex & $\begin{array}{l}\mathrm{SL} \\
(\mathrm{cm})\end{array}$ & $\begin{array}{l}\text { Sexual } \\
\text { maturity }\end{array}$ & $\begin{array}{l}\text { Cranial } \\
\text { maturity }\end{array}$ \\
\hline \multicolumn{8}{|l|}{ Skull } \\
\hline $\begin{array}{l}\text { Crassicauda sp. lesions in pterygoids, frontals, left } \\
\text { palatine and right alisphenoid }\end{array}$ & $\mathrm{ACO}-17$ & 29/5/98 & Playa Chucho & indet & 145 & (imm) & imm \\
\hline $\begin{array}{l}\text { Crassicauda sp. lesions in pterygoids, left frontal and } \\
\text { right palatine }\end{array}$ & JAS-26 & $25 / 10 / 93$ & Chancay & indet & indet & indet & imm \\
\hline $\begin{array}{l}\text { Crassicauda sp. lesions in pterygoids, right alisphenoid } \\
\text { and right frontal }\end{array}$ & KVW-2381 & 15/1/93 & Pacasmayo & indet & indet & indet & imm \\
\hline Crassicauda sp. lesions in left pterygoid & ACO-21 & 29/5/98 & Lagunilla & indet & indet & indet & imm \\
\hline Crassicauda sp. lesions in both pterygoids & LAS-5 & 6/8/99 & Salaverry & indet & indet & indet & indet \\
\hline Crassicauda sp. lesions in right pterygoid & JAS-175 & 27/11/99 & Sechura & indet & indet & indet & indet \\
\hline Crassicauda sp. lesions in right pterygoid & MFB-770 & 22/1/99 & Puerto Rico & indet & indet & indet & indet \\
\hline Crassicauda sp. lesions in pterygoids & MWC-26 & $15 / 12 / 87$ & Peru & indet & indet & indet & mat \\
\hline Crassicauda sp. lesions in left pterygoid and left exoccipital & MFB-159 & $21 / 4 / 93$ & Chimbote & $\mathrm{F}$ & 228 & mat & mat \\
\hline Crassicauda sp. lesions in pterygoids & KVW-2425 & 5/11/95 & Pimentel & indet & indet & indet & mat \\
\hline Crassicauda sp. lesions in pterygoids & AGG-619 & 22/10/92 & Huarmey & indet & indet & indet & mat \\
\hline Crassicauda sp. lesions in pterygoids & KVW-2382 & 17/1/93 & Santa Rosa & indet & indet & indet & mat \\
\hline Crassicauda sp. lesions in left frontal & KVW-2426 & $5 / 11 / 95$ & Santa Rosa & indet & indet & indet & mat \\
\hline Crassicauda sp. lesions in left maxillary and right pterygoid & MFB-137 & $15 / 4 / 93$ & Cerro Azul & M & 237.5 & mat & mat \\
\hline Crassicauda sp. lesions in left pterygoid & KVW-643 & $27 / 7 / 87$ & Pucusana & $\mathrm{F}$ & 215 & (mat) & mat \\
\hline Crassicauda sp. lesions in left pterygoid & KVW-2423 & $5 / 11 / 95$ & San Jose & indet & indet & indet & mat \\
\hline Crassicauda sp. lesions in left pterygoid & MFB-142 & $15 / 4 / 93$ & Cerro Azul & M & 226.5 & mat & mat \\
\hline Crassicauda sp. lesions in left pterygoid & MFB-109 & $30 / 3 / 93$ & Cerro Azul & M & 231 & mat & mat \\
\hline Crassicauda sp. lesions in pterygoids & KVW-2399 & 25/10/93 & Chancay & indet & indet & indet & mat \\
\hline Crassicauda sp. lesions in right pterygoid & KVW-2000 & 27/12/89 & Sechura & indet & indet & indet & mat \\
\hline Crassicauda sp. lesions in right pterygoid & KVW-2391 & 22/1/93 & Besique & indet & 236 & (mat) & mat \\
\hline Crassicauda sp. lesions in right pterygoid & RBC-17 & 26/3/93 & Chimbote & M & 240 & mat & mat \\
\hline Crassicauda sp. lesions in right pterygoid & KVW-2403 & $31 / 5 / 94$ & Pucusana & $\mathrm{M}$ & 241 & mat & mat \\
\hline Crassicauda sp. lesions in pterygoids & MFB-250 & $13 / 6 / 93$ & Cerro Azul & indet & indet & indet & $\mathrm{imm}$ \\
\hline Congenital malformation of the skull & JCR-1351 & $15 / 4 / 88$ & Pucusana & $\mathrm{F}$ & 207 & $\mathrm{imm}$ & mat \\
\hline Crest on the rostrum & MFB-191 & $13 / 5 / 93$ & Cerro Azul & $\mathrm{F}$ & 211.5 & mat & mat \\
\hline $\begin{array}{l}\text { Healed fracture of left mandible; Crassicauda sp. } \\
\text { lesions in left pterygoid }\end{array}$ & MFB-741 & $13 / 1 / 95$ & Matacaballo & indet & indet & indet & mat \\
\hline Osteolysis in left maxillary & $\mathrm{RBC}-19$ & 26/3/93 & Chimbote & M & 241.5 & mat & mat \\
\hline $\begin{array}{l}\text { Osteolysis in maxillaries and right premaxillary; } \\
\text { Crassicauda sp. lesions in pterygoids }\end{array}$ & JAS-17 & $24 / 6 / 93$ & Pucusana & M & 247.5 & mat & mat \\
\hline Osteolysis in the left maxillary & MFB-174 & $-/ 4 / 93$ & Cerro Azul & indet & indet & indet & mat \\
\hline Osteolysis of the distal extremity of the premaxillaries & MFB-756 & 16/7/98 & Pucusana & indet & indet & indet & imm \\
\hline Osteolysis of the right maxillary & AGG-621 & $26 / 10 / 92$ & Casma & indet & indet & indet & mat \\
\hline $\begin{array}{l}\text { Osteomyelitis and osteolysis of left pterygoid and } \\
\text { palatinum }\end{array}$ & KVW-2401 & $-/ 4 / 94$ & Chancay & indet & indet & indet & mat \\
\hline Osteomyelitis and osteolysis of left mandible & KVW-2400 & 25/10/93 & Chancay & indet & indet & indet & mat \\
\hline Slight lateral deviation of the snout & RBC-21 & 26/3/93 & Chimbote & M & 234 & mat & mat \\
\hline Traumatic lesions in the occipital & KVW-994 & $13 / 12 / 87$ & Pucusana & M & 229 & $\mathrm{imm}$ & mat \\
\hline \multicolumn{8}{|l|}{ Head, trunk and appendages } \\
\hline Chronic fibrotic reaction on tail stock and kyphosis & AGG-405 & $5 / 9 / 91$ & Ancon & $\mathrm{F}$ & 167.5 & $\mathrm{imm}$ & indet \\
\hline Deformation of the backbone & KVW-1426 & 16/6/88 & Pucusana & $\mathrm{F}$ & 171.5 & (imm) & indet \\
\hline Deformation of the dorsal fin & KVW-582 & 19/6/87 & Pucusana & M & 244 & mat & indet \\
\hline Healed fracture of right mandibular ramus & KVW-522 & $11 / 1 / 87$ & Pucusana & $\mathrm{F}$ & 184.5 & (imm) & imm \\
\hline Healed lesions of the rostrum & MFB-189 & $13 / 5 / 93$ & Cerro Azul & M & 232 & mat & mat \\
\hline Brachygnathia & MFB-220 & $15 / 5 / 93$ & Cerro Azul & $\mathrm{F}$ & 202 & indet & indet \\
\hline Insertion of flipper broken & RBC-21 & 26/3/93 & Chimbote & M & 234 & mat & mat \\
\hline Insertion of flipper broken & $\mathrm{RBC}-22$ & $26 / 3 / 93$ & Chimbote & $\mathrm{F}$ & 233 & mat & mat \\
\hline Mastitis & KVW-523 & $11 / 1 / 87$ & Pucusana & $\mathrm{F}$ & 191 & $\mathrm{imm}$ & indet \\
\hline Nodule on tail stock & KVW-2403 & $31 / 5 / 94$ & Pucusana & M & 241 & mat & mat \\
\hline \multicolumn{8}{|l|}{ Genital tract } \\
\hline Chronic orchitis & KVW-2403 & $31 / 5 / 94$ & Pucusana & M & 241.0 & mat & mat \\
\hline Ovarian cysts & MFB-191 & $13 / 5 / 93$ & Cerro Azul & $\mathrm{F}$ & 211.5 & mat & mat \\
\hline Vesicular lesions on the penis & MFB-510 & $18 / 5 / 94$ & Cerro Azul & M & 207 & imm & indet \\
\hline Ucerated lesion on the penis & MFB-675 & $9 / 7 / 94$ & Cerro Azul & M & 200.5 & imm & indet \\
\hline
\end{tabular}




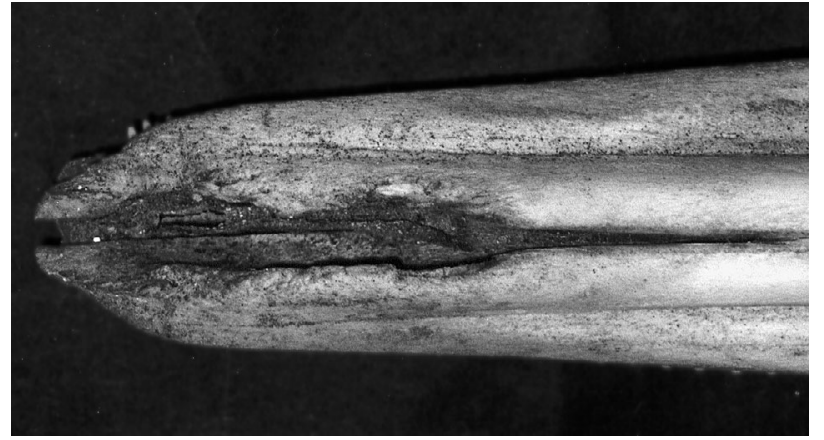

Fig. 5. Delphinus capensis. Osteolysis of the distal extremities of the premaxillaries in a cranially immature dolphin of indeterminate sex (MFB-756)

was $10 \mathrm{~mm}$ shorter than the maxillaries and premaxillaries (brachygnathia). The tooth rows, especially on the maxillaries, were abnormally oriented outward.

The skull of a mature male (RBC-21) showed a slight lateral deviation at the distal extremity of the mandible. In a mature female (MFB-191), an unusual $50 \times 3 \times 2 \mathrm{~mm}$ crest was present on the distal half of the left maxillary.

Traumatic lesions. In a mature specimen (MFB-741), the thickened proximal extremity of the left mandibular ramus stood out ventrally from the surrounding bone and presented a dark lateral line, presumably of re-ossification. The ramus apparently had suffered a fracture that subsequently healed.

Two holes (diameters 15 and $5 \mathrm{~mm}$ ) with irregular edges, likely inflicted by a blunt object, perforated the occipital bone close to the left condyle in a mature male (KVW-994, Fig. 7), harpooned off central Peru.

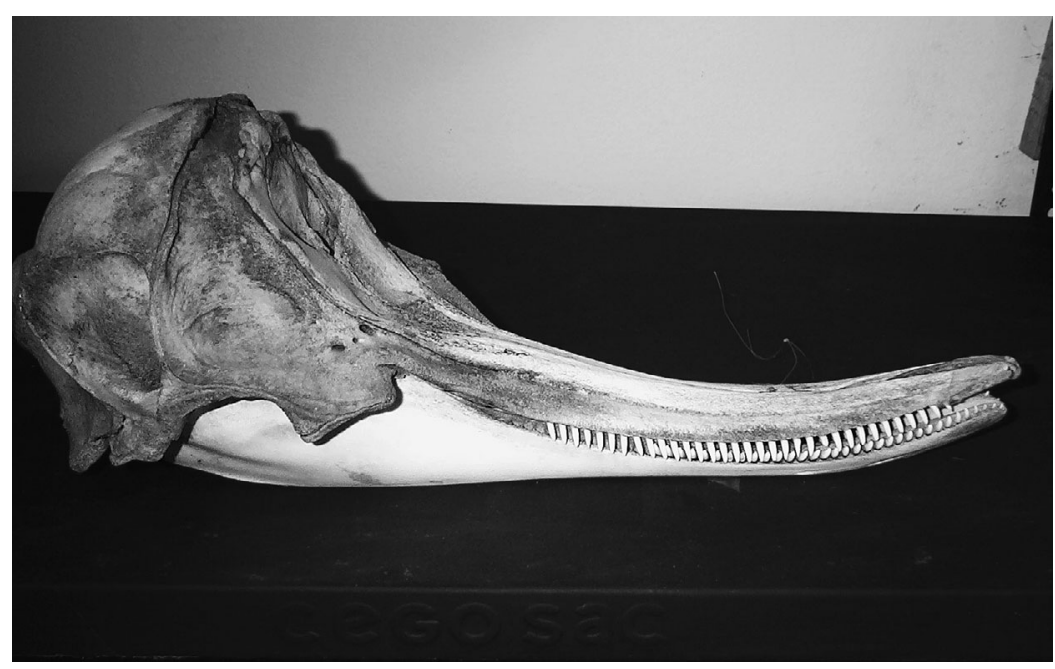

Fig. 6. Delphinus capensis. Congenital malformation of the rostrum in cranially mature female JCR-1351

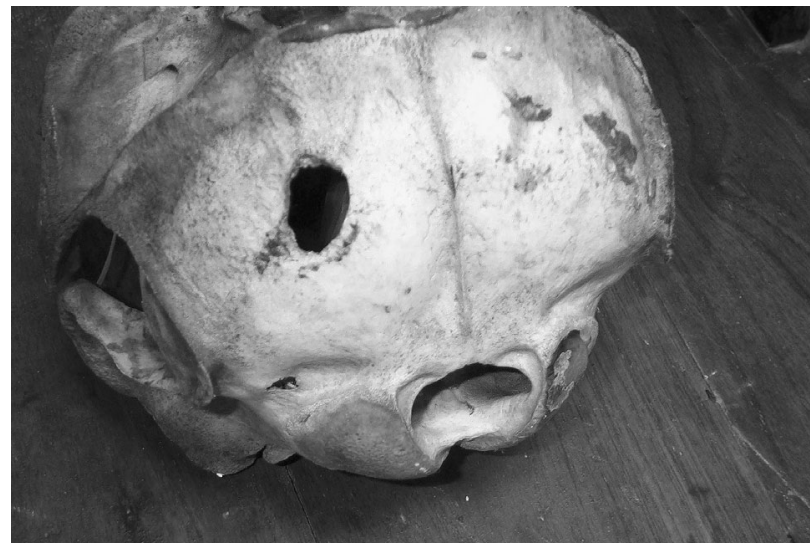

Fig. 7. Delphinus capensis. Two abnormal holes with irregular edges in the occipital bone of mature male KVW-994

Epidemiology. Cranial lesions and abnormalities (excluding alveoli and teeth) were observed in 36 of 103 dolphins $(35 \%)$. The skulls of the 4 cranially immature individuals were normal. Prevalences of malformations and traumas were 2.9 and $1.9 \%$, respectively. Lytic lesions, including those caused by Crassicauda sp. and those associated with osteomyelitis, occurred in 32 of 103 dolphins $(31.1 \%)$ and accounted for $84.2 \%$ of all injuries. Osteolysis and osteomyelitis occurred as frequently in cranially adult females $(22.2 \%, \mathrm{n}=9)$ as in males $(22.6 \%, \mathrm{n}=31)$ and therefore sexes were pooled. Prevalence of these lesions was similar $\left(\chi^{2}=0.331,1 \mathrm{df}\right.$, $\mathrm{p}=0.56)$ in adults $(33.8 \%, \mathrm{n}=68)$ and immatures $(27.3 \%, \mathrm{n}=22)$. Crassicauda sp. cranial bone damage was diagnosed in $26.5 \%$ of skulls $(\mathrm{n}=98)^{\mathbf{1}}$. There was no significant difference (Fisher's, $p=0.8$ ) in prevalence of crassicaudiasis between cranially adult females $(25 \%, \mathrm{n}=8)$ and males $(19.4 \%, \mathrm{n}=31)$, allowing pooling of sexes. Prevalence of Crassicauda cranial bone damage was similar $\left(\chi^{2}=0.177,1 \mathrm{df}, \mathrm{p}=0.67\right)$ in cranially immature $(22.7 \%, \mathrm{n}=22)$ and adult $(27.3 \%$, n = 66) dolphins. Crassicauda sp. infestation caused $78.8 \%$ of the observed lytic lesions ( $n=33$ ).

Dental and periodontal diseases. Teeth were broken or damaged in 9 individuals ( 7 adults, 2 of unknown maturity). Four of these also showed lesions of the alveoli. In a large, cra-

\footnotetext{
1 The presence/absence of Crassicauda sp. lesions could not be ascertained in 5 of the 103 skulls; hence, they were excluded from the statistical analysis
} 
nially adult male (ACO-63), 23 teeth were severely worn and broken.

One to 4 sets of 'paired teeth' ( 2 teeth, typically of unequal size, in parallel at a single alveolus locus) were observed on both maxillaries in 2 of 10 freshly dead dolphins that were landed together (Table 3). Two paired teeth were also present on the right maxillary of an adult skull but not in cranial material of 21 other dolphins (Table 3). The alveoli of 16 dolphins were enlarged or partially/totally filled by new, cancellous bone formation. Both maxillaries and mandibles were affected. The number of occluded alveoli per individual varied from 1 to 60 .

Dental and periodontal infections were responsible for at least $6.1 \%$ of all the lytic lesions of the skull. Acquired tooth lesions were found in $39.1 \%$ of specimens $(n=23)$, paired teeth in $9.4 \%(n=32)$. Partially or fully occluded alveoli were observed in 16 of 103 $(15.5 \%)$ dolphins. Fourteen were cranially adult, while the maturity of the 2 others was unknown. Prevalence of lesions of the alveoli was $20 \%$ in 70 cranially mature skulls. Two of $9(22.2 \%)$ adult females and 11 of 32 $(34.4 \%)$ adult males were affected.

\section{Lesions of the head, trunk and appendages}

\section{Subsample A.}

Head backbone and appendages: Serious injuries and deformations of the backbone, dorsal fin and head were observed in 3 of 314 (0.95\%) long-beaked common dolphins (Table 4). The distal end of the right mandibular ramus showed a healed fracture in an immature female (KVW-522). A mature male (KVW582) had a seriously twisted dorsal fin, and an immature female (KVW-1426) suffered scoliokyphosis, a posterior and laterally deformed spine.

Mastitis: A nodule was sampled from tissues associated with the mammaries of an immature female (KVW-523). Microscopic findings included inflammation of the acinar and ductular tissue associated with diffuse, focally severe lympho-plasmacytic infiltrate. Several large granulomas contained hyaline material and numerous giant cells. The lesion was diagnosed as chronic mastitis and could have been caused by parasites (e.g. Crassicauda sp.) or a bacterial infection. However, no evidence of parasitism was found.

\section{Subsample B.}

Backbone and appendages: Injuries and externally visible deformations of the spine and tailstock were only observed in 2 of $545(0.37 \%)$ dolphins (Table 4$)$. An immature female (AGG-405) with severe kyphosis at thorax height also had a large nodule on the left side of the tailstock. Microscopic examination of the module revealed a subcutaneous mass of fibrous tissue containing a sparse infiltrate of inflammatory cells. Some muscle tissue was caught up in the general inflammatory reaction and fibrosis, but there was no evidence of any 'primary' muscle disease. The nodule was diagnosed as a chronic fibrotic reaction due to an infection or a trauma. This animal was also afflicted by a severe poxvirus infection as revealed by an unusually high density of 'tattoo' skin lesions spread over its entire body. The second case, a mature male (KVW2403), had a large nodule on the right side of the tailstock, with a large, lytic lesion affecting at least 1 caudal vertebra. The same animal was ill with chronic orchitis (see section 'Genital lesions' below).

Severely torn tissues, apparently with dislocated glenoid articulation in at least 1 flipper, in 2 mature dolphins (RBC-21 and -22) landed together, were presumably the result of the traumatic net-entanglement that caused their death.

Rostrum: Fibrous tissue $(5 \mathrm{~mm})$ protruded from the left distal extremity of the beak as well as from the left mouth gape in an adult male (MFB-189). We believe these were healed lesions. A female of unknown sexual maturity (MFB-220) had brachygnathia and also presented another congenital malformation, namely 4 sets of paired teeth on both maxillaries.

\section{Cutaneous lesions}

Tattoo skin lesions.

Subsample C: Tattoo skin lesions were distributed over the entire body of a cranially immature female (KVW-276). Caught off Pucusana in January 1986, this individual represents the earliest confirmed report of tattoo skin disease in cetaceans from the eastern South Pacific.

Subsample D: Tattoos were seen in 13 ( 7 males, 6 females) of 27 dolphins (48.2\%) taken off Ancon in 1991 and 1992. All were sexually immature.

Subsample E: The epidemiology of tattoo skin disease in 46 Delphinus capensis of this subsample was reported in Van Bressem \& Van Waerebeek (1996).

\section{Punctiform marks.}

Subsample D: Dark grey or black points perceptible to the touch, with or without a pit in the centre (Fig. 8) were observed in 12 ( 7 females, 5 males) of 27 (44.4\%) dolphins examined. All were immature. The marks were restricted to the head in 3 cases and generalised in the others. Poxvirus particles were found by TEM (D. Dekegel and G. Van Heule, pers. comm. to M.F.B, May 1991) in samples of skin marks, described in field notes as 'tenuous points with a faint depression', scattered over the whole body of male AGG-573, which did not show typical tattoos. Punctiform marks on the other dolphins were not examined by TEM. 
Subsample E: Punctiform marks were reported in 3 of $56(5.35 \%)$ dolphins landed at Cerro Azul and Culebras. The positive specimens included 2 males and a female, all immature.

\section{Round marks.}

Subsample D: Many tenuous, light grey, round marks, up to $40 \mathrm{~mm}$ in diameter and distributed over the whole body, were observed in an immature male (AGG-567, Fig. 9a). Examination by TEM revealed

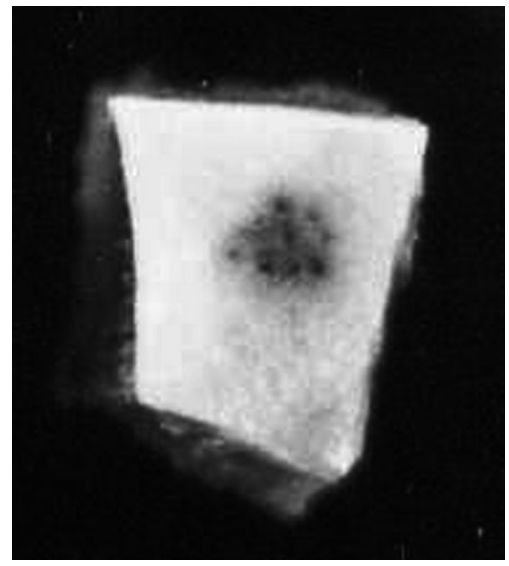

Fig. 8. Lagenorhynchus obscurus. Punctiform marks sampled in a Peruvian dusky dolphin (AGG-577), showing skin with identical pathomorphism diagnosed as punctiform marks in Delphinus capensis in the present paper

a

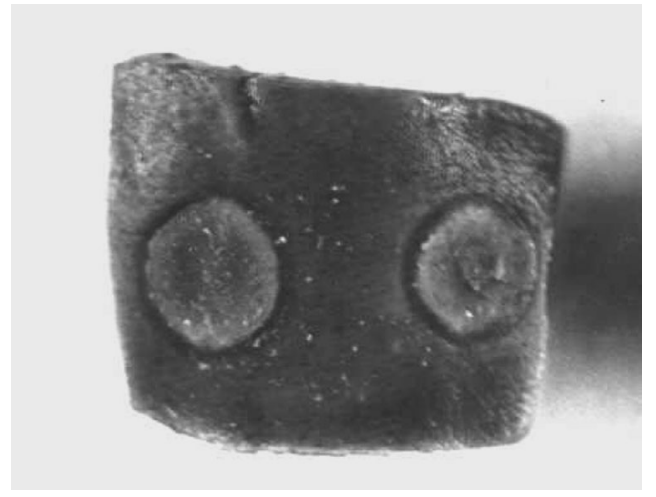

b

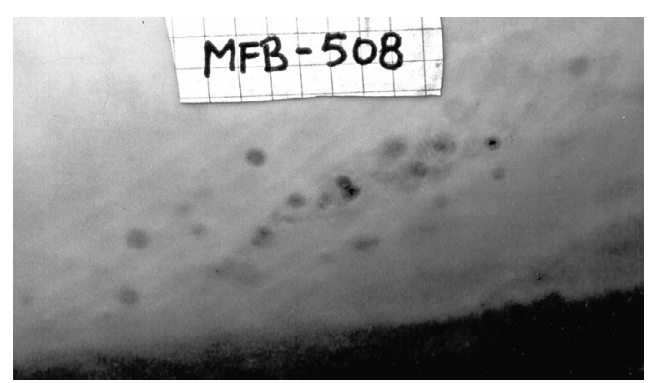

Fig. 9. Delphinus capensis. (a) Round skin marks sampled in immature male AGG-567. (b) Round skin marks on the belly of immature male MFB-508 unidentified parasites and viral particles (D. Dekegel and G. Van Heule, pers. comm. to M.F.B, May 1991).

Subsample E: A few (1 to 5) to many (20 to 30) of light to dark grey marks up to $10 \mathrm{~mm}$ in diameter were observed ventrally and on the flanks of 7 of 56 specimens (12.5\%, Fig. 9b). All affected dolphins were males and 2 of them were sexually mature.

\section{Dark circle lesions.}

Subsample E: A few (1 to 5) to many (more than 20) dark circles measuring about $2 \mathrm{~mm}$ in diameter dotted the belly and flanks of 5 of $56(8.93 \%)$ dolphins. The skin inside the circles was of the same colour as the healthy skin. The affected animals included 2 adults and 3 immatures, all males. One adult (MFB-229) showed a very high number of dark circle lesions, a low density of tattoo marks and was noticeably thin. Interestingly, 3 of the affected dolphins were landed on the same day.

Coronet marks.

Subsample E: One to 3 marks that appeared as rounded crowns, hence referred to as coronet marks, were found on the belly of a female of unknown sexual maturity and an immature male caught off Cerro Azul in 1993. Prevalence of coronet marks was 3.6\% $(n=56)$.

Scars.

Subsample C: Large scars were seen on the head of a male (KVW-546) and a female (KVW-522), both sexually immature. A white area $(3.5 \mathrm{~cm})$ underside of the left fluke in another immature female (JCR-1573) may represent a scar or a discoloration of unknown aetiology.

Subsample E: Whitish-grey scars that had likely not been caused by bites or tooth rakes from conspecifics or other large animals were observed in 15 of $54^{2}$ $(27.8 \%)$ dolphins. All but 1 male were sexually immature. Prevalence of scars was not significantly different $\left(\chi^{2}=0.5, \mathrm{df}=1, \mathrm{p}=0.48\right)$ between females $(36.4 \%, \mathrm{n}=$ 11 ) and males $(25.6 \%, \mathrm{n}=43)$. Only a few (1 to 4$)$ of these scars were present on the head, flanks, flippers or tailstock. They measured between $28 \times 15 \mathrm{~mm}$ and $85 \times 30 \mathrm{~mm}$. A small abscess was associated with 1 scar in an immature female. In 11 of the 15 dolphins, or $20.4 \%$ of the long-beaked common dolphins examined for scars, the appearance, size and location suggested that the scars were the remnants of wounds inflicted during interactions with fisheries.

\section{Anomalous pigmentation.}

Subsample E: The distal extremity of the left flipper of an immature male (MFB-675) bore small white spots of unknown origin, although the skin looked otherwise smooth and healthy. Prevalence of this type of anomalous pigmentation was $1.8 \%(\mathrm{n}=56)$.

\footnotetext{
${ }^{2}$ Two dolphins of Subsample E were not examined for scars
} 


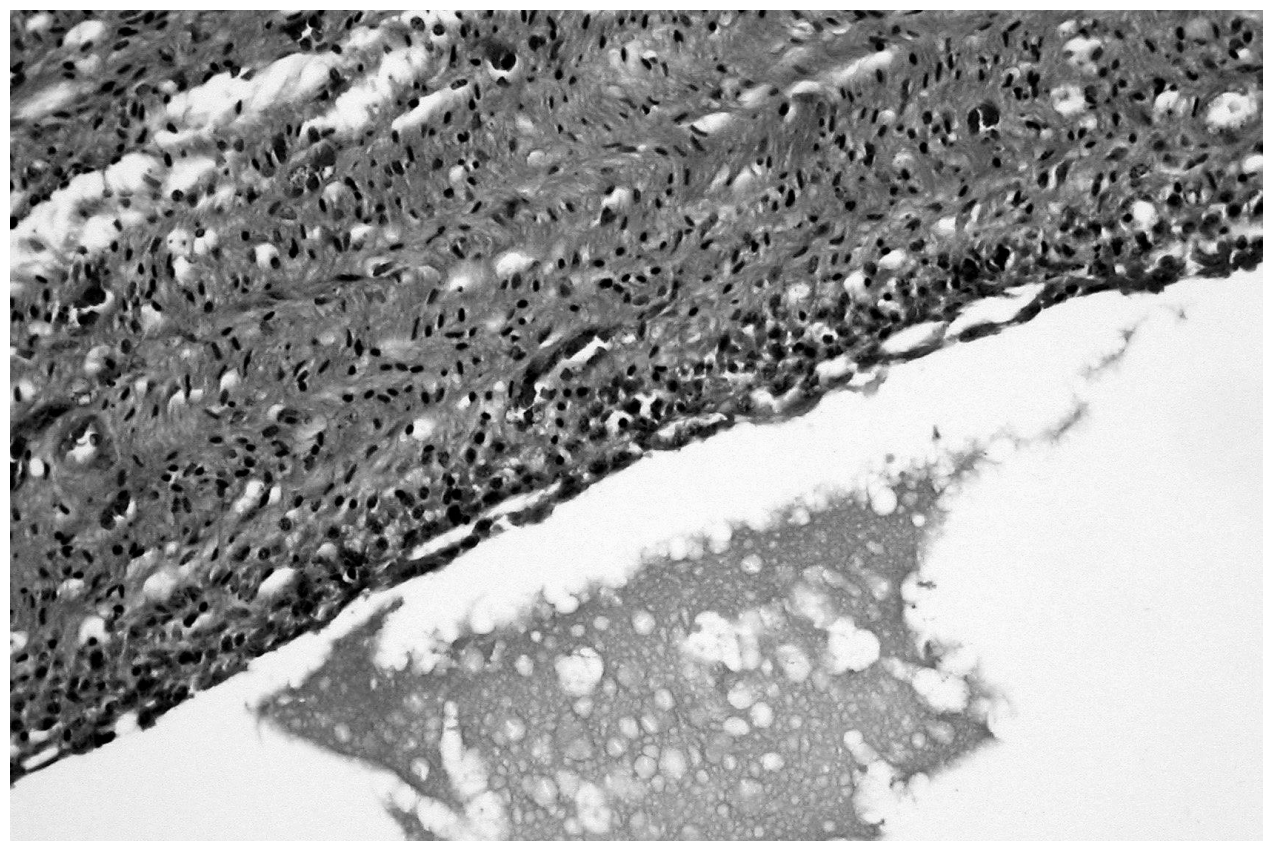

Fig. 10. Delphinus capensis. Follicular cyst in the ovary of lactating dolphin MFB-191. Cyst is lined by a thin layer of granulosa cells and contains proteinaceous fluid

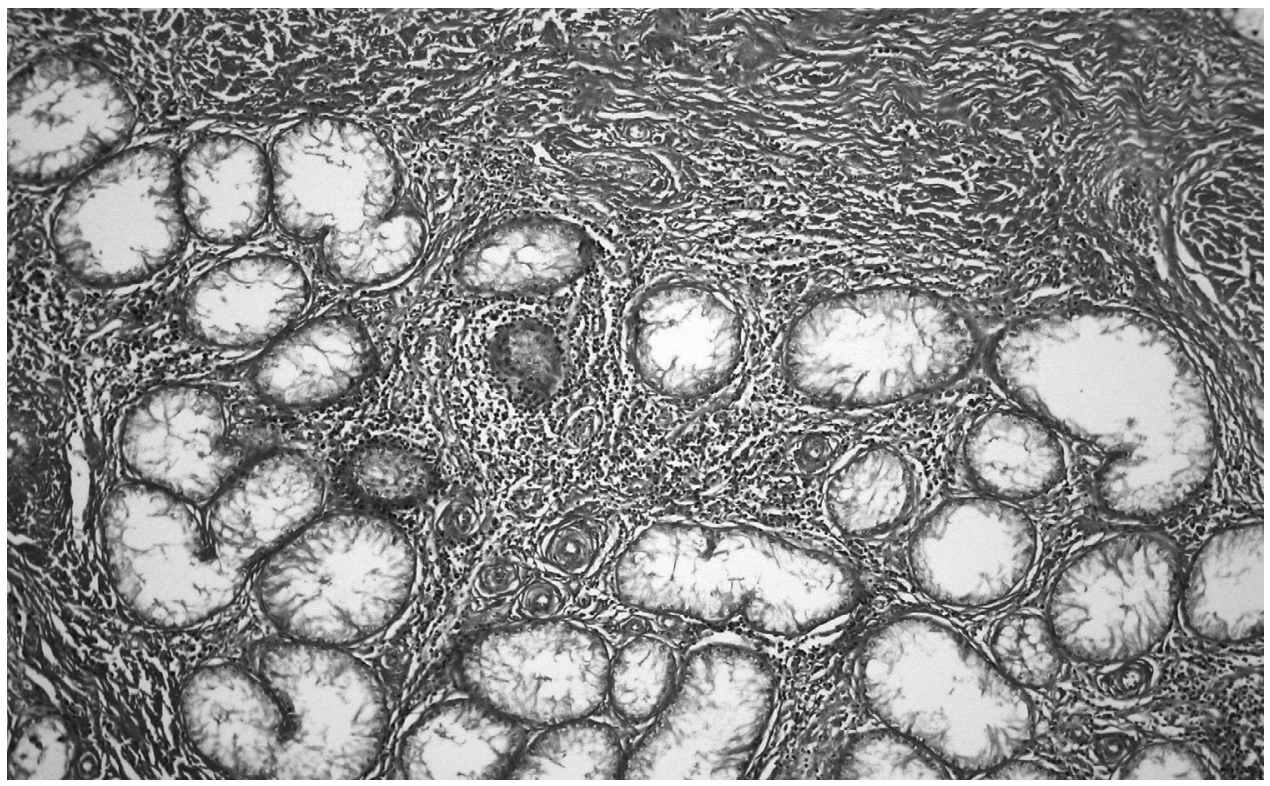

Fig. 11. Delphinus capensis. Orchitis in adult male KVW-2403. There is a degeneration of the seminiferous tubules, mononuclear cell infiltration and fibroplasia

\section{Genital lesions}

Subsample F.

Ovarian cysts: A large cyst $(19 \times 17 \times 15 \mathrm{~mm})$ projected from the left ovary of a lactating dolphin (MFB-191, Table 4). When the ovary was sliced, several smaller cysts were detected, all of which contained a gelatinous material. A corpus luteum $(10 \times$
$10 \mathrm{~mm}$ ) contained a similar gelatinous mass. Histological findings included the presence of several cysts of variable size lined by thin layers of epithelium with abundant basophilic cytoplasm (Fig. 10) which were suggestive of follicular cysts. No abnormal cystic structures were noted in the ovaries of 23 other females. Prevalence of ovarian cysts in this sample was $4.2 \%$. 


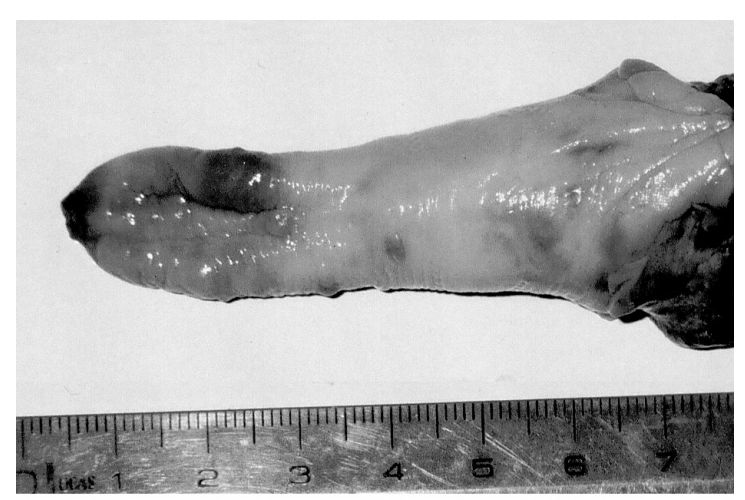

Fig. 12. Delphinus capensis. Several congested zones and some small vesicles on the penis of immature dolphin MFB-510. Ruler in cm

Orchitis: Two abscesses affected the right testis of a sexually mature male (KVW-2403) (Table 4). Microscopic changes in a sample of 1 abscess included marked fibroplasia and mild diffuse lympho-plasmacytic inflammatory cell infiltration (Fig. 11). Many seminiferous tubules were degenerated, or necrotic and mineralised. Degenerate and necrotic tubules contained large numbers of macrophages and neutrophils. A few necrotic ductules were mineralised. The lesion was diagnosed as chronic orchitis with severe fibroplasia. This dolphin also had a hard mass on the right side of its tailstock (see above). Prevalence of orchitis was $1.3 \%$ in this sample $(\mathrm{n}=78)$.

Vesicular lesions of the penis: Several congested zones and some small vesicles were present on the penis of an immature dolphin (MFB-510, Fig. 12). A white and ulcerated vesicle was visible at the basis of the penis of another immature animal (MFB-675, Table 4). Macroscopically, the vesicles did not resemble the genital warts previously described in this species (Van Bressem et al. 1996) but rather resembled genital herpesvirus lesions. Prevalence of penile vesicular lesions was $16.7 \%(\mathrm{n}=12)$.

Subsample G. There was no mention of lesions of the genital tract in the available database relating to 65 female and 61 male Delphinus capensis.

\section{DISCUSSION}

This study revealed miscellaneous lesions, diseases and malformations of the skull, teeth, head, trunk, appendages, skin and genital tract of Delphinus capensis from Peru. The highest prevalence of lesions was observed in the teeth $(39.1 \%)$, skull (35\%), penis $(16.7 \%)$ and skin (up to $48.2 \%$, depending on the type). Forty-two dolphins had at least 2 types of injuries that affected 1 or more organs. Multiple lesions of various organs have been described in the harbour porpoise Phocoena phocoena, short-beaked common dolphin D. delphis, striped dolphin Stenella coeruleoalba, common bottlenose dolphin Tursiops truncatus and Risso's dolphin Grampus griseus from British waters (Baker 1992, Baker \& Martin 1992) as well as in belugas Delphinapterus leucas from the St Lawrence estuary (De Guise et al. 1995), to cite only a few.

Osteolysis was the most common lesion in the skull of Delphinus capensis. Infestation by Crassicauda sp. and tooth infections were responsible for 78.8 and $6.1 \%$ of observed osteolysis, respectively, the remainder being of unknown aetiology. Adult roundworms Crassicauda spp. infest the cranial sinuses of several species of small cetaceans, including D. capensis from Peruvian waters (Dailey 1985, K. Van Waerebeek et al. 1994 unpubl.) and produce the typical (apparently irreversible) perforating lytic bone lesions with a basket-like appearance that often deform pterygoids (Raga et al. 1982, Dailey 1985). In D. capensis, prevalence of Crassicauda sp. related skull damage was $26.5 \%$. This was lower than the prevalence observed in offshore common bottlenose dolphins (68.8\%, $\mathrm{n}=16$ ) occuring sympatrically but higher than the prevalence in a large sample of dusky dolphins Lagenorhynchus obscurus from Peru and Chile (0.37\%, n = 267, Van Waerebeek et al. 1990, 1993) and a smaller, more recent sample from Peru $(4.3 \%$ $\mathrm{n}=46$, Montes-Iturrizaga 2003). Interestingly, prevalence of Crassicauda sp. associated bone damage did not vary with cranial maturity status in D. capensis, and extensive lesions were observed in both mature and immature specimens. In spotted dolphins Stenella attenuata from the Eastern Tropical Pacific, prevalence was higher in younger animals, which was attributed to mortality caused by Crassicauda sp. infestation in young dolphins (Perrin \& Powers 1980). Crassicaudiasis may not cause significant mortality in D. capensis. The lower prevalence of live worms $(8 \%, \mathrm{n}=25)$ than bone lesions $(26.5 \%, \mathrm{n}=98)$ suggests that dolphins may recover from infestation but remain marked (Van Waerebeek et al. 1994). The percentage of extensive lesions seemed higher in immature $(60 \%, \mathrm{n}=5)$ than in mature $(22.2 \%, \mathrm{n}=18)$ dolphins. No other worms have been associated with basket-like cranial bone lesions in cetaceans. Besides, Stenurus sp., another nematode that commonly infests the air sinuses of Delphinidae, was not found in D. capensis examined during this study (CEPEC unpubl. data).

Adult dolphins showed a high prevalence of worn and broken teeth as well as alveolar lesions. The latter are likely a consequence of tooth decay and loss (De Smet 1977). The loss of a large number of teeth with resulting damage to the alveoli and, eventually, lysis of 
surrounding bone tissue, as seen in some dolphins (JAS-17 and RBC-19), may have caused considerable pain. De Smet (1977) reported that tooth lesions were common in a sample of 12 Tursiops truncatus from various ocean provinces and from captivity. Worn and missing teeth were also described in 5 of 32 (juveniles and adults) Phocoena phocoena from British waters (Baker \& Martin 1992) and in at least 15 of 24 belugas from the St Lawrence estuary (De Guise et al. 1995). Periodontal disease is known from common bottlenose dolphin, dusky dolphin, short-finned pilot whale Globicephala macrorhynchus, long-finned pilot whale G. melas, Risso's dolphin, and Burmeister's porpoise P. spinipinnis from Peruvian waters (Montes-Iturrizaga 2003) as well as in belugas (De Guise et al. 1995). The presence of paired teeth in 2 dolphins landed together suggests that they were genetically related.

Cranial bone fractures were only found in the mandible of 1 of 75 complete skulls. This injury may have been inflicted by conspecific or interspecific interactions, or by fishing gear. Mandible fractures have been described in Phocoena phocoena and Tursiops truncatus from the North Sea as well as in $T$. truncatus, Globicephala macrorhynchus and Lagenorhynchus obscurus from the SE Pacific (van Bree \& Duguy 1970, Montes-Iturrizaga 2003). Prevalence varied between $3.2 \%$ in $31 \mathrm{~L}$. obscurus and $7.3 \%$ in $55 \mathrm{~T}$. truncatus from Peru (Montes-Iturrizaga 2003).

Other traumas of the skull, body and skin in several specimens of Peruvian Delphinus capensis were likely caused by fishery interactions. In dolphin KVW-994, cranial perforations were possibly inflicted by blows to the head. The dolphin had been harpooned and was likely clubbed to death in the boat, a common fate of small cetaceans caught alive in Peru (K. Van Waerebeek, L. Chavez-Lisambart and I. Garcia-Godos unpubl. data). Two dolphins (RBC-21, RBC-22) presumably hurt their flippers while attempting to escape the nets that eventually killed them. Dolphins KVW-522 and MFB-189 likely escaped an earlier capture event but not without injuring their head. One female (AGG-405) showed a chronic subcutaneous fibrotic reaction on the tailstock, possibly due to a netcaused trauma. In at least another 11 dolphins caught off central Peru in 1993 and 1994, large scars observed on the trunk, appendages and head were thought to be remnants of harpoon wounds or selfinflicted injuries in the struggle to free themselves from fishing devices. White-grey scars on the back of several D. capensis caught off Ancon in 1991 and 1992 were believed to be healed harpoon wounds (I. Garcia-Godos unpubl. data). Severe traumas due to fishing devices likely resulted in secondary mortality of unassessed numbers of injured dolphins that managed to escape. Therefore, total fisheries-related dolphin mortality is thought to be higher than can be accounted for by the tallying of landed specimens. In Peru, long-beaked common dolphins were frequently captured by industrial purse-seiners, including directed sets, at least until 1994.

Although malformations of the skull and trunk were found in several individuals, their prevalence in the population was low. The most striking malformation was the, likely congenital, curvature of the rostrum of female JCR-1351. While this may have reduced this individual's ability to catch prey, the deformation was evidently viable. Other less spectacular congenital malformations of the skull and teeth were presumed to be of even less consequence to survival. It is unknown whether malformations of the dorsal fin and backbone were congenital or acquired. Deformities of the dorsal fin and backbone are known from the killer whale Orcinus orca, common dolphin (likely Delphinus delphis), Tursiops truncatus and Hector's dolphin Cephalorhynchus hectori (Wilson et al. 1997, Visser 1998, Berghan \& Visser 2000 ). Classification and possible origins of backbone deformities in cetaceans are discussed in Berghan and Visser (2000).

Chronic mastitis, observed in 1 pubescent female, possibly arose from a parasitic (e.g. Crassicauda sp.) or bacterial infection. Acute and chronic mastitis are known from at least 6 other odontocetes including Delphinus delphis, Atlantic white-sided dolphin Lagenorhynchus acutus, Stenella coeruleoalba, Tursiops truncatus, Delphinapterus leucas, and Globicephala melas but not D. capensis (Sweeney \& Ridgway 1975, Geraci et al. 1978, Raga \& Balbuena 1993, Kuiken et al. 1994, De Guise et al. 1995, Di Guardo et al. 1995). The disease was caused by Crassicauda spp. in L. acutus and G. melas, while Aeromonas hydrophila and Edwardsiella tarda were isolated in a D. leucas and T. truncatus, respectively. Crassicauda spp. have not been extracted from the mammary glands of Peruvian small cetaceans, but very few mammaries have been examined in detail (Van Waerebeek unpubl. data).

Ovarian cysts (possibly follicular cysts) were found in a lactating female. Prevalence of ovarian cysts was $4.2 \%$ ( $\mathrm{n}=24)$, similar to that observed in Lagenorhynchus obscurus from the same region $(3.06 \%, \mathrm{n}=98$; Van Bressem et al. 2000). Ovarian cysts, including follicular and luteinized cysts, have also been reported from L. obliquidens, Delphinapterus leucas and Stenella coeruleoalba (Harrison et al. 1972, De Guise et al. 1995, Munson et al. 1998). The aetiology of follicular cysts is not known in dolphins. In cattle, aberration of the preovulatory surge of luteinizing hormone, either the absence or mistiming of the surge, is thought to cause this condition (McEntee 1990, Kennedy \& Miller 1993).

The chronic orchitis in 1 adult male (KVW-2403) may be the origin of the bone lesions afflicting several cau- 
dal vertebrae. Bacterial and fungal diseases of the urinary tract and testes are common sources of infection in animals suffering vertebral osteomyelitis (Kornegay \& Barber 1980). Interestingly, infection by Brucella spp. may lead to vertebral osteomyelitis in humans and dogs (Kornegay \& Barber 1980, Rajapakse 1995) as well as to orchitis in mammals. Brucellae are known to circulate among Peruvian Delphinus capensis (Van Bressem et al. 2001a) and may be a plausible cause for the orchitis and the vertebral lesions in KVW-2403. Among small cetaceans, orchitis was described only from the Amazon river dolphin Inia geoffrensis and Tursiops truncatus (Simpson \& Gardner 1972, Sweeney \& Ridgway 1975). Vertebral osteomyelitis was reported from a captive $T$. truncatus (Alexander et al. 1989).

The aetiology of vesicular lesions of the penis in 2 immature Delphinus capensis is unknown, but herpesviruses are possible agents. Members of the Alphavirinae subfamily cause vesicles, pustules and shallow ulcers in the genital tract of humans, bovines and horses (Whitley 1990, Fenner et al. 1993). Herpesviruses were also briefly reported in lesions of the cervix and penis of harbour porpoises (Ross et al. 1994).

Besides tattoo skin lesions reported in the present paper and in Van Bressem \& Van Waerebeek (1996), we encountered several other skin defects, most, however, of unknown aetiology. Poxvirus particles were detected by TEM in punctiform marks possibly analogous with ring lesions, the early form of tattoos (Geraci et al. 1979). In cetaceans, poxvirus particles have always been associated with tattoo and ring lesions (Flom \& Houk 1979, Geraci et al. 1979, Van Bressem et al. 1993a). Tattoos and punctiform marks occurred together in some specimens (see Table 3). Herpes-like virus particles were demonstrated in skin marks also described as 'black points' on the rostrum of 2 Lagenorhynchus obscurus (Van Bressem et al. 1994) and may also have caused some of the punctiform marks in Delphinus capensis. Conceivably, lesions of at least 2 different aetiologies were included under the term 'punctiform marks' in 1991 and 1992, partly accounting for the difference in prevalence between Subsamples D (44.4\%) and E (5.35\%). Round marks were detected in Subsamples D and E. Unidentified parasites and virus particles were visible by TEM in samples taken from 1 dolphin. Additional samples should be studied by this technique in order to determine the aetiology of skin marks other than tattoos. The location, appearance, density and epidemiological features of the round marks and dark circles described in Subsample E suggest that they may be the same lesions at different developmental stages. Anomalous pigmentation seen in 1 dolphin was possibly due to piebaldness, a genetic melanisation defect also known as partial albinism (Comings \& Odland 1966, Van Waerebeek 1992). Prevalence of this defect was low $(1.8 \%)$. Anomalous pigmentation (including melanistic and all white coloration) was seen in $6.4 \%$ of 358 photo-identified common dolphins Delphinus sp. from Hauraki Gulf, New Zealand (Stocking \& Visser 2005).

We conclude that long-beaked common dolphins from the Southeast Pacific are affected by a variety of acquired, congenital, traumatic, infectious and parasitic diseases. Some of these are severe and bound to impair normal vital functions and behaviour. Of all diseases encountered, morbillivirus, poxvirus and Brucella sp. infections, as well as Crassicauda sp. infestation, appear to have the highest potential for significant adverse impact on population abundance by increasing natural mortality and/or by negatively affecting reproduction (Perrin \& Powers 1980, Van Bressem et al. 1999). Interactions with artisanal and industrial fisheries on Peru's continental shelf are responsible for the large majority of human-induced mortality, and are thought to be the principal cause of debilitating physical traumas in this dolphin population. The feasibility of applying fishing gear modifications and other potential by-catch mitigation measures, including regulatory instruments, should be re-evaluated in the region as one of the most relevant issues for the enhanced conservation of this marine mammal population.

Acknowledgements. Financial support for data analysis and reporting was provided by the Small Cetacean Voluntary Fund of the International Whaling Commission, drawn from a contribution to the Fund by the Humane Society of the United States (HSUS). Field work in Peru from 1985 to 2000 was supported by many organisations, the principal sponsors including the Gesellschaft zum Schutz der Meeressäugetiere, Belgian Agency for Developing Aid, Leopold III Fonds voor Natuuronderzoek en Natuurbehoud, United Nations Environment Programme (UNEP), International Fund for Animal Welfare (IFAW), International Union for Conservation of Nature and Natural Resources (IUCN)/Cetacean Specialist Group, Whale and Dolphin Conservation Society, Cetacean Society International and Chicago Zoological Society. We warmly thank Dr. Dekegel and G. Van Heule for TEM analysis, Luis Santillan and Ruth Bello for contributing samples, and Diana Vega for histology of gonads. We are grateful to 2 anonymous referees for critically reviewing the manuscript and offering valuable suggestions.

\section{LITERATURE CITED}

Alexander JW, Solangi MA, Riegel LS (1989) Vertebral osteomyelitis and suspected diskospondylitis in an Atlantic bottlenose dolphin (Tursiops truncatus). J Wildl Dis 25:118-121

Baker JR (1992) Causes of mortality and parasites and incidental lesions in dolphins and whales from British waters. Vet Rec 130:569-572 
Baker JR, Martin AR (1992) Causes of mortality and parasites and incidental lesions in harbour porpoises (Phocoena phocoena) from British waters. Vet Rec 130: 554-558

Benirschke K, Henderson JR, Sweeney JC (1984). A vaginal mass, containing fetal bones, in a common dolphin, Delphinus delphis. Rep Int Whaling Comm Spec Iss 6: 457-458

Berghan J, Visser IN (2000) Vertebral column malformations in New Zealand delphinids with a review of cases world wide. Aquat Mamm 26:17-25

Birkun A, Kuiken T, Krivokhizhin S, Haines DM, Osterhaus ADME, Van de Bildt MWG, Joiris CR, Siebert U (1999) Epizootic of morbilliviral disease in common dolphins (Delphinus delphis ponticus) from the Black Sea. Vet Rec 144:85-92

Comings DE, Odland GF (1966) Partial albinism. JAMA 195: 519-523

Cowan DF, Walker WA, Brownell RL (1986) Pathology of small cetaceans stranded along southern California beaches. In: Bryden MM, Harrison R (eds) Research on dolphins. Oxford University Press, Oxford, p 323-367

Dailey MD (1985). Diseases of Mammalia: Cetacea. In: Kinne O (ed) Diseases of marine mammals, Vol 4, Part 2. Biologische Anstalt Helgoland, Hamburg, p 805-847

De Guise S, Lagace A, Beland P, Girard C, Higgins R (1995) Non-neoplastic lesions in beluga whales (Delphinapterus leucas) and other marine mammals from the St. Lawrence Estuary. J Comp Pathol 112:257-271

De Smet WMA (1977) The fate of old bottle-nosed dolphins, Tursiops truncatus, in nature as revealed by the condition of their skeletons. Aquat Mamm 5 (3):78-86

Di Guardo G, Agrimi U, Morelli L, Cardeti G, Terracciano G, Kennedy S (1995) Post mortem investigations on cetaceans found stranded on the coasts of Italy between 1990 and 1993. Vet Rec 136:439-442

Dixon, JM (1984) Hepatitis and bone lesions in a stranded juvenile comon dolphin Delphinus delphis Linnaeus. Aust Mamm 7:225-228

Duignan PJ, House C, Geraci JR, Duffy N and 7 others (1995) Morbillivirus infection in cetaceans of the western Atlantic. Vet Microbiol 44:241-249

Fenner FJ, Gibbs EPJ, Murphy FA, Rott R, Studdert MJ, White DO (1993) Veterinary virology, 2nd edn. Academic Press, San Diego, CA, p 666

Flom JO, Houk EJ (1979) Morphologic evidence of poxvirus in 'tattoo' lesions from captive bottlenosed dolphins. J Wildl Dis 15:593-596

Flom JO, Brown RJ, Jones RE (1978) Fibrous osteodistrophy in a wild dolphin. JAVMA. 173:1124-1126

Geraci JR, Lounsbury VJ (1993) Marine mammals ashore, a field guide for strandings. Texas A\&M University Sea Grant College Program, TX, p 182-184

Geraci JR, Dailey MD, St Aubin DJ (1978) Parasitic mastitis in the Atlantic white-sided dolphin Lagenorhynchus acutus, as a probable factor in herd productivity. J Fish Res Board Can 35:1350-1355

Geraci JR, Hicks BD, St Aubin DJ (1979) Dolphin pox: a skin disease of cetaceans. Can J Comp Med 43:399-404

Harrison RJ, Brownell RL, Boice RC (1972) Reproduction and gonadal appearance in some odontocetes. In: Harrison RJ (ed) Functional anatomy of marine mammals, Vol I. Academic Press, London, p 361-429

Hohn AA, Chivers SJ, Barlow J (1985) Reproductive maturity and seasonality of male spotted dolphins, Stenella attenuata, in the eastern tropical Pacific. Mar Mamm Sci 1: 273-293
Jepson PD, Brew S, MacMillan AP, Baker JR and 5 others (1997) Antibodies to Brucella in marine mammals around the coast of England and Wales. Vet Rec 141:513-515

Kennedy PC, Miller RB (1993) The female genital system. In: Jubb KVF, Kennedy PC, Palmer N (eds) Pathology of domestic animals. Academic Press, London, p 349-469

Kornegay JN, Barber DL (1980) Diskospondylitis in dogs. JAVMA 15:337-341

Kuiken T, Simpson VR, Allchin CR, Bennett PM and 8 others (1994) Mass mortality of common dolphins (Delphinus delphis) in south west England due to incidental capture in fishing gear. Vet Rec 134:81-89

López A, Benavente A (1993) Calculo vaxinal nun golfiño comun (Delphinus delphis, L.) varado en Galicia. Eubalaena $2: 3-7$

McEntee K (1990) Cysts in and around the ovary. In: McEntee K (ed) Reproductive pathology of domestic mammals. Academic Press, London, p 52-68

Montes-Iturrizaga D (2003) Descripción y evaluación de alteraciones anatómicas óseas cráneo-mandibulares en cetáceos odontocetos del mar peruano. Lic thesis, National University of San Marcos, Lima

Munson L, Calzada N, Kennedy S, Sorensen TB (1998) Luteinized ovarian cysts in Mediterranean striped dolphins. J Wildl Dis 34:656-660

Osterhaus ADME, Broeders HWJ, Teppema JS, Kuiken T, House JA, Vos HW, Visser IKG (1993) Isolation of virus with rhabdovirus morphology from a white-beaked dolphin (Lagenorhynchus albirostris). Arch Virol 133: 189-193

Perrin WF, Powers JE (1980) Role of a nematode in natural mortality of spotted dolphins. J Wildl Manag 44:960-963

Perrin WF, Coe JM, Zweifel JR (1976) Growth and reproduction of the spotted porpoise, Stenella attenuata, in the offshore Eastern Tropical Pacific. Fish Bull 74:229-269

Raga JA, Balbuena JA (1993) Parasites of the long-finned pilot whale Globicephala melas (Traill, 1809) in European waters. Rep Int Whaling Comm Spec Iss 14:391-406

Raga JA, CasinosA, Filella S, Raduan MaA (1982) Notes on cetaceans of the Iberian coasts. V. Crassicauda grampicola Johnston \& Mawson, 1941 (Nematoda) cause of injuries in the pterygoids of some specimens of Grampus griseus. Saeugetierkd Mitt 30:315-318

Rajapakse CN (1995) Bacterial infections: osteoarticular brucellosis. Bailliere's Clin Rheumatol 9:161-177

Reidarson TH, McBain J, House C, King DP and 5 others (1998) Morbillivirus infection in stranded common dolphins from the Pacific Ocean. J Wildl Dis 34:771-776

Ross HM, Reid RJ, Howie FE, Gray EW (1994) Herpesvirus infection of the genital tract in harbour porpoise Phocoena phocoena. Proc 9th Annu Conf Eur Cetacean Soc, Montpellier, France, March 1994. European Cetacean Society, Lugano

Ross HM, Jahans KL, MacMillan AP, Reid RJ, Thompson PM, Foster G (1996) Brucella species infection in North Sea seal and cetacean populations. Vet Rec 138:647-648

Sanino GP, Van Waerebeek K, Yáñez J (2003a) Revisión de la distribución del genero Delphinus y registros documentados de Delphinus capensis, en Chile. Bol Mus Nac Hist Nat Chile 52:97-102

Sanino GP, Hamilton-West C, Rojas A, Yañez JL, Van Waerebeck K (2003b). Estudios de restos varados de Delphinus delphis, y primer registro documentado de Pneumonia focal abscedativa, en Chile. Bol Mus Nac Hist Nat Chile 52:103-117 (in Spanish)

Sawyer JE, Walker WA (1977) Vaginal calculi in the dolphin. J Wildl Dis 13:346-348 
Simpson JG, Gardner MB (1972) Comparative microscopic anatomy of selected marine mammals. In: Ridgway SH (ed) Marine mammals of the sea, biology and medicine. Charles C. Thomas, Springfield, IL, p 363-377

Stocking KA, Visser IN (2005) Anomalously pigmented common dolphins (Delphinus sp.) off northern New Zealand. Aquat Mamm 31:43-51

Sweeney JC, Ridgway SH (1975) Common diseases of small cetaceans. JAVMA 167:533-540

Swinscow TDV (1981) Statistics at square one, 7th edn. British Medical Association, London

Thrusfield M (1986) Veterinary epidemiology. Butterworths \& Co, London, p 280

Van Bree PJH, Duguy R (1970) Sur quelques abérrations pathologiques chez les petits Cétacés. Zool Gart 39:11-15

Van Bressem MF, Van Waerebeek K (1996) Epidemiology of poxvirus in small cetaceans from the Eastern South Pacific. Mar Mamm Sci 12:371-382

Van Bressem MF, Van Waerebeek K, Reyes JC, Dekegel D, Pastoret PP (1993a) Evidence of poxvirus in dusky dolphin (Lagenorhynchus obscurus) and Burmeister's porpoise (Phocoena spinipinnis) from coastal Peru. J Wildl Dis 29: 109-113

Van Bressem MF, Visser IKG, De Swart RL, Orvell C, Stanzani L, Androukaki E, Siakavara K, Osterhaus ADME (1993b) Dolphin morbillivirus infection in different parts of the Mediterranean sea. Arch Virol 129:235-242

Van Bressem MF, Van Waerebeek K, Garcia-Godos A, Dekegel D, Pastoret PP (1994) Herpes-like virus in dusky dolphins Lagenorhynchus obscurus, from coastal Peru. 1994. Mar Mamm Sci 10:354-359

Van Bressem MF, Van Waerebeek K, Piérard G, Desaintes C (1996) Genital and lingual warts in small cetaceans from coastal Peru. Dis Aquat Org 26:1-10

Van Bressem MF, Van Waerebeek K, Fleming M, Barrett T (1998a) Serological evidence of morbillivirus infection in small cetaceans from the Southeast Pacific. Vet Microbiol 59:89-98

Van Bressem MF, Jepson P, Barrett T (1998b) Further insight on the epidemiology of cetacean morbillivirus in the Northeastern Atlantic. Mar Mamm Sci 14:605-613

Van Bressem MF, Van Waerebeek K, Raga JA (1999) A review of virus infections of cetaceans and the potential impact of morbilliviruses, poxviruses and papillomaviruses on host population dynamics. Dis Aquat Org 38: 53-65

Van Bressem MF, Van Waerebeek K, Siebert U, Wünschmann A, Chávez-Lisambart L, Reyes JC (2000) Genital diseases in the Peruvian dusky dolphin (Lagenorhynchus obscurus). J Comp Pathol 122:266-277

Van Bressem MF, Van Waerebeek K, Raga JA, Godfroid J, Brew SD, MacMillan AP (2001a) Serological evidence of Brucella species infection in odontocetes from the south Pacific and the Mediterranean. Vet Rec 148:657-661

Van Bressem MF, Montes D, Van Waerebeek K, Reyes JC, Ontón K, Alfaro-Shigueto J, Garcia-Godos A (2001b) Bone lesions and abnormalities of the skulls of long-snouted common dolphins (Delphinus capensis) from Peru.

Editorial responsibility: Murray Dailey, Sausalito, California, USA
Abstracts of the 14th Biennal Conf Biol Mar Mamm, 28 Nov-3 Dec 2001 Vancouver, Canada

Van Bressem MF, Van Waerebeek K, Jepson PD, Raga JA and 13 others (2001c) An insight into the epidemiology of dolphin morbillivirus worldwide. Vet Microbiol 81:287-304

Van Waerebeek K (1992) Population identity and general biology of the dusky dolphin Lagenorhynchus obscurus (Gray, 1828) in the Southeast Pacific. PhD thesis, University of Amsterdam

Van Waerebeek K (1993) Geographic variation and sexual dimorphism in the skull of the dusky dolphin, Lagenorhynchus obscurus (Gray, 1828). Fish Bull 91:754-774

Van Waerebeek K, Reyes JC (1994) Post-ban small cetacean takes off Peru: a review. Rep Int Whaling Comm Spec Iss ue 15:503-520

Van Waerebeek K, Reyes JC, Read AJ, McKinnon JS (1990) Preliminary observations of bottlenose dolphins from the Pacific coast of South America. In: Leatherwood S, Reeves RR (eds) The bottlenose dolphin. Academic Press, San Diego, CA, p 143-154

Van Waerebeek K, Reyes JC, Alfaro J (1993) Helminth parasites and phoronts of dusky dolphins Lagenorhynchus obscurus (Gray, 1828) from Peru. Aquat Mamm 19: 159-169

Van Waerebeek K, Van Bressem MF, Reyes IC, Garćia-Godos A, Alfaro I, Ontón K, Bello R, Echegaray M (1994) Illegal exploition of small cetaceans in Peru. Final report to the United Nations Environment Programme (UNEP), Nairobi and to the Dolphin Conservation Society, Bath, p 76

Van Waerebeek K, Van Bressem MF, Felix F, Alfaro J, and 5 others (1997) Mortality of dolphins and porpoises in coastal fisheries off Peru and southern Ecuador in 1994. Biol Conserv 81:43-49

Van Waerebeek K, Van Bressem MF, Alfaro-Shigueto J, Sanino GP, Montes D, Ontón K (1999) A preliminary analysis of recent captures of small cetaceans in Peru and Chile. Document SC/51/SM, 17 May 1999, Scientific Committee of the International Whaling Commision, Grenada

Van Waerebeek K, Alfaro-Shigueto J, Montes D, Onton K, Santillan L, Van Bressem MF, Vega D (2002) Fisheries related mortality of small cetaceans in neritic waters of Peru in 1999-2001. Document SC/54/SM10, 26 April-10 May 2002, Scientific Committee of the International Whaling Commision, Shimonoseki

Visser IKG, Van Bressem MF, De Swart RL, van de Bildt MWG and 8 others (1993) Characterization of morbilliviruses isolated from dolphins and porpoises in Europe. J Gen Virol 74:631-641

Visser IN (1998) Prolific body scars and collapsing fins on killer whales (Orcinus orca) in New Zealand waters. Aquat Mamm 24:71-81

Wilson B, Thompson PM, Hammond PS (1997) Skin lesions and physical deformities in bottlenose dolphins in the Moray Firth: population prevalence and age-sex differences. Ambio 26:243-247

Whitley RJ (1990) Herpes simplex viruses. In: Fields BN, Knipe DM, Howley PM (eds) Virology. Raven Press, New York, p 1843-1887

Submitted: January 30, 2005; Accepted: July 19, 2005

Proofs received from author(s): December 9, 2005 